\title{
¿Se puede saber si una función tiene primitiva elemental?
}

\author{
Walter Mora F. \\ wmora2@itcr.ac.cr \\ Escuela de Matemática \\ Instituto Tecnológico de Costa Rica
}

Resumen. Este artículo presenta una introducción elemental a la teoría de Lioville (en versión antigua y moderna) y un teorema de Chebyshev, sobre la integración en términos finitos. Se presenta la parte histórica, la parte computacional y la parte algebraica.

Palabras clave: Primitivas, funciones elementales, integración en términos finitos

Abstract. This article presents an elementary introduction to Lioville theory (in old version and modern version) and also a Chebyshev theorem, on integration in finite terms. It is presented the historical part, the computational part and the algebraic part.

KeyWords: Primitive, elementary functions, integration in finite terms

\subsection{Introducción}

En los cursos de cálculo elemental, el problema de cálculo de integrales consiste en: "dada una función elemental $f$, calcular otra función elemental (si existe) $g$ tal que $g^{\prime}=f$ (o $\int f=g$ )". La solución parcial de este problema se presenta como un proceso heurístico que emplea una "caja de trucos" (tablas de integrales, sustituciones, integración por partes y descomposición en fracciones parciales). Solo en el caso de funciones racionales $P / Q$ aparece un algoritmo finito (fracciones parciales). Los métodos empleados no son métodos de decisión, solo de cálculo: Si no hay éxito en el cálculo de la primitiva de 
una de una función elemental $f$, no se sabe si esta primitiva se puede expresar o no, en términos de funciones elementales.

Si uno observa varios ejemplos de cálculo de primitivas, no parece que hubiera un patrón regular entre el integrando y la antiderivada.

\section{Ejemplo 1.1}

Por ejemplo, usando Wolfram Mathematica obtenemos,

$\operatorname{In}[]:=$

$$
\begin{array}{ll}
\text { Integrate }\left[1 /\left(\mathbf{x}^{\wedge} \mathbf{2}+\mathbf{1}\right), \mathbf{x}\right] & (*=\operatorname{ArcTan}[x] *) \\
\text { Integrate[1/Sqrt } \left.\left[1-\mathbf{x}^{\wedge} 2\right], \mathbf{x}\right] & (*=\operatorname{ArcSin}[x] *) \\
\text { Integrate[Log[x+Sqrt } \left.\left.\left[\mathbf{x}^{\wedge} 2-1\right]\right], \quad \mathbf{x}\right] & \left(*=-\sqrt{-1+x^{2}}+x \log \left[x+\sqrt{-1+x^{2}}\right] *\right)
\end{array}
$$

Sin embargo, si introducimos logaritmos y el número $i$ se puede empezar a ver un patrón que, como vamos a ver, es persistente.

\section{Ejemplo 1.2}

Usando Wolfram Mathematica obtenemos,

$\operatorname{In}[]:=$

$$
\begin{aligned}
& \text { Integrate }\left[1 /\left(\mathbf{x}^{\wedge} 2+1\right), \mathbf{x}\right] \quad\left(*=\frac{1}{2} i \log (x+i)-\frac{1}{2} \log (x-i) \quad *\right) \\
& \text { Integrate[1/Sqrt } \left.\left[1-\mathbf{x}^{\wedge} 2\right], \mathbf{x}\right] \quad\left(*=-i \log \left(\sqrt{1-x^{2}}+i x\right)\right. \\
& \text { Integrate }\left[\log \left[\mathbf{x}+\operatorname{Sqrt}\left[\mathbf{x}^{\wedge} 2-1\right]\right], \quad \mathbf{x}\right] \quad\left(*=-\sqrt{-1+x^{2}}+x \log \left[x+\sqrt{-1+x^{2}}\right] *\right)
\end{aligned}
$$

J. Liouville fue el primero que probó un teorema que permite tener una base teórica para decidir si una función elemental (las funciones usuales del cálculo y otras más) tiene primitiva elemental basado en la forma que debería tener la función y su primitiva. 
En general, si $F$ es una función algebraica con primitiva elemental, entonces bajo algunas condiciones se tiene,

$$
\int F\left(x, y_{1}, y_{2}, \ldots, y_{n}\right) d x=V_{0}+\sum_{j=1}^{n} c_{j} \log \left(V_{j}\right)
$$

Aquí, $F\left(x, y_{1}, y_{2}, \ldots, y_{n}\right)$ es una expresión algebtraica $F\left(x, \log x, \mathbb{e}^{x}, \ldots\right)$.

Por medio de este principio, Liouville pudo probar que varias familias de funciones no tienen primitiva elemental, en especial ciertas integrales elípticas, que era uno de los temas que dominaba el universo matemático de su tiempo.

Este teorema tiene su generalización en términos puramente algebraicos, sigue dominado la forma en la que deben aparecer las primitivas elementales en el caso general. Sorpresivamente, el algoritmo para la integración de funciones racionales $P / Q$ que veremos más adelante, es similar al que se usa en el caso general.

Organización. Este artículo se divide en dos partes. En la primeria parte vamos a ver los teoremas de integración en términos finitos que fueron establecidos en el siglo XIX.

a) El algoritmo de J. Bernoulli (1703) que es el método de integración por descomposición en fracciones parciales que se enseña todavía en cálculo. Este algoritmo tiene valor teórico solamente.

b) El teorema de Chebyshov (1853) que nos permite decidir de manera fácil si funciones de la forma $x^{m}\left(a+b x^{n}\right)^{p}$ con $a, b, n, m, p \in \mathbb{Q}$, tienen primitiva elemental o no.

c) El teorema (fuerte) de Liouville y dos casos especiales, para aplicarlo a ciertas familias de funciones (la teoría la desarrollo Lioville entre 1833 y 1841).

En la segunda inicia con el algoritmo para integrar funciones racionales (método de Hermite y el algoritmo de Rothstein/Trager) pues esta es una guía para los otros algoritmos. Para considerar una introducción algoritmo general, se incluye una introducción mínima al Álgebra diferencial y el teorema de Liouville desde esta perpectiva. Se hace la prueba de un caso especial del teorema de Liouville (solo un caso, porque la maquinaria algebraica es muy extenuante y a veces muy avanzada). La segunda parte estará dedicada a una introducción mínima al algoritmo de Risch; este un algoritmo general para calcular primitivas o decidir si la primitiva no es elemental. Solo consideramos el algoritmo para casos de funciones con extensiones logarítmicas.

En general, solo necesitamos hacer cálculos con álgebra de polinomios: División de polinomios con resto, mcd de dos polinomios y el algoritmo extendido de Euclides. Como la perpectiva es computacional, todos los ejemplos de prueba para los algoritmos se harán con Wolfram Mathematica.

\subsection{Primitivas en general}


Para efectos computacionales, una función $y=f(x)$ tiene primitiva si existe una función $g(x)$ tal que $g^{\prime}(x)=f(x)$. Como es usual, se escribe

$$
\int f(x) d x=g(x)+\text { constante }
$$

\section{Interludio: Primitivas en análisis matemático}

¿Toda función tiene una primitiva?. Aunque estamos acostumbrados a una clase de funciones que si tiene primitiva, en realidad las primitivas son escasas (en el mismo sentido que "casi" no hay racionales en $\mathbb{R}$ ). Históricamente la integración fue definida como el proceso inverso de la derivada, es decir, la integral de $f$ es entendida como una función $F$ tal que $F^{\prime}=f$. Desde el punto de vista del análisis matemático y algunas aplicaciones en física e ingeniería, esto es muy limitado porque hay funciones que no son una derivada (de otra función). Las funciones continuas sin son derivadas (de alguien), pero las funciones con "discontinuidades de salto" no. Alrededor de 1850 A. Cauchy y B. Riemman construyen un concepto de integral independiente del concepto de derivada, usando la noción de "área bajo la curva". Finalmente un función acotada en $[a, b]$ es integrable en el sentido Riemann si su conjunto de discontinuidades tiene medida cero (un conjunto numerable de discontinuidades). Hay funciones $f$ cuya derivada $f^{\prime}$ tienen una cantidad no numerable de discontinuidades, así que " $\int f^{\prime}=f^{\prime \prime}$ no aplica, al menos para la integral de Riemann (en otra noción de integral, llamada "generalizada de Riemann", esta identidad si aplica [1, Sección 8.1]).

Una vez que la derivada y la integral han sido definidas de manera independiente, el "Teorema fundamental del cálculo" revela la relación inversa entre ambos conceptos. En realidad nos interesa "el segundo" teorema fundamental del cálculo: $\operatorname{Si} f:[a, b] \rightarrow \mathbb{R}$ es integrable (en el sentido Riemann), entonces $F(x)=\int_{a}^{x} f(x) d x$ es continua y si $f$ es continua en $x=c \in[a, b]$, entonces $F^{\prime}(c)=f(c)$.

Como las funciones continuas son integrables, tenemos: Si $f:[a, b] \rightarrow \mathbb{R}$ es continua, entonces $f$ tiene una primitiva (es derivada de alguien). Si $x \in[a, b]$, la función $F(x)=\int_{a}^{x} f(x) d x$ es derivable y satisface $F^{\prime}(x)=f(x)$ en $[a, b]$.

No toda función es derivada (de alguien). El teorema del valor intermedio para derivadas (Teorema de Darboux) dice que una función con una 'discontinuidad de salto' no puede ser derivada de otra función. Por ejemplo, la función $f(x)=\operatorname{signo}(\mathbf{x})$ toma los valores $-1,0,1$ si $x<0, x=0$ o $x<0$, respectivamente; como el conjunto de discontinuidades es finito, es Riemann integrable, pero no es derivada de otra función. Esta es una de las bondades de que la integral no dependa de la derivada.

Como una curiosidad adicional, las funciones continuas tienen primitiva... pero la mayoría de funciones continuas no tienen derivada en ningún punto!. Raro?. Esto es una consecuencia del "Teorema de Categoría de Baire". Desde el punto de vista de este teorema, 
los conjuntos "flacos" son los conjuntos "nunca densos" (su complemento es denso). Los conjuntos que son unión contable de este tipo de conjuntos se llama "magro" o de "primera categoría". Los conjuntos de segunda categoría son los que no son de primera categoría, son los conjuntos "gordos", como $\mathbb{R}$. Resulta que las funciones continuas que son derivables al menos en un punto es un conjunto de "primera categoría", es decir, las funciones diferenciables son excesivamente atípicas en general ([1, Sección 8.2]), algo como lo que pasa con los familiares números racionales y los irracionales en $\mathbb{R}$. Como consecuencia, las primitivas son funciones bastante raras.

Desde el punto de vista computacional, un comando como "Integrate $[f, x]$ " devuelve una función (si tiene éxito) $g$ tal que $g^{\prime}=f$, y en general no se especifica el dominio. Como es sabido, las primitivas de $f$ difieren solo en una constante y pueden tener aspecto diferente, así que pueden aparecer diferencias de apariencia.

\section{Ejemplo 1.3}

Vamos a usar MAтнемAтіcA para calcular algunas primitivas. Este software asume que algunas funciones son de variable compleja por lo que a veces hay que especificar que la variable $x$ es real.

a) Primitiva de $f(x)=\frac{1}{x}$ (en el software $\log [x]$ corresponde a $\ln x$ ).

$$
\begin{array}{ll}
\operatorname{In}[]:=\operatorname{Integrate}[1 / x, x] & \operatorname{In}[]:=D[\log [x], x] \\
\text { Out }[]=\log [x] & \text { Out }[]=1 / x
\end{array}
$$

b) También podemos calcular $g(x)=\int_{-1}^{x} \frac{1}{t} d t$

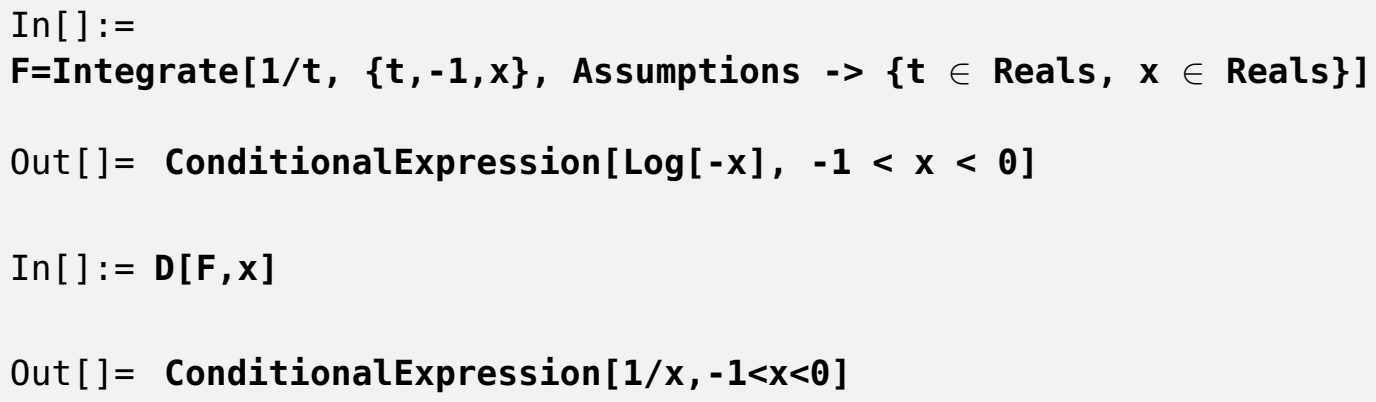




\subsection{Primitivas elementales}

Para efectos computacionales, una función $f$ tiene primitiva elemental si existe una función $g$ elemental tal que $g^{\prime}=f$. Como es usual, se escribe

$$
\int f(x) d x=g(x)+\text { constante }
$$

Las funciones "elementales" son las funciones del cálculo usual: Funciones racionales, funciones algebraicas (explícitas e implícitas), exponenciales y logarítmicas (estas son las "trascendentales elementales") y las fuciones definidas por uan combinación finita de estas tres clases anteriores.

Por ejemplo, $\sqrt{\tan \left(\mathbb{e}^{x^{2}}\right)-\ln (1+\sqrt{x})}+x^{x}+\ln (\operatorname{arcsen} x)$ es una función elemental. La expresión $x^{x}$ se interpreta como $\mathbb{e}^{x \ln x}$.

Las funciones racionales se definen como el resultado de un número finito de adiciones y multiplicaciones en la variable $x$ y pueden ser expresadas como un cociente de polinomios en $x$ (con exponentes enteros y coeficientes en $\mathbb{R}$ o $\mathbb{C}$ ). Por ejemplo, $\frac{1+\mathbb{e}^{x}}{1-2 e^{2 x}}$ es una función racional de $\mathbb{e}^{x}$ y $\frac{1+\mathbb{e}^{-x}}{1+3 \mathbb{e}^{2 x}}$ es una función racional de $\mathbb{e}^{x}$ si se reescribe como $\frac{1+\mathbb{e}^{x}}{e^{x}+3 \mathbb{e}^{3 x}}$.

Las funciones algebraicas son funciones " $y$ " que satisfacen una ecuación del tipo

$$
y^{n}+f_{n-1} y^{n-1}+\ldots .+f_{0}=0
$$

donde cada $f_{i}$ es una función racional. Por ejemplo, $y=\sqrt{x}+\sqrt{x+\sqrt{x}}$ es algebraica porque satisface la ecuación $y^{4}-4 x y^{2}-4 x y-x=0$.

Esta "definición" de función elemental es suficiente para los propósitos de este artículo.

Si admitimos funciones de variable compleja, las funciones elementales se reducen a funciones racionales, exponenciales y logarítmicas y las combinaciones algebraicas mencionadas. Sea $z=x+\boldsymbol{i} y$. La exponencial compleja se define como

$$
\mathbb{e}^{z}=\mathbb{e}^{x}(\cos y+i \operatorname{sen} y) \text { para todo } z \in \mathbb{C}
$$

Si $x=0$ tenemos, $\mathbb{e}^{i y}=\cos y+i \operatorname{sen} y, y$ de aquí deducimos sen $y=\frac{\mathbb{e}^{i y}-\mathbb{e}^{-i y}}{2 i}$ y $\cos y=\frac{\mathbb{e}^{i y}+\mathbb{e}^{-i y}}{2}$

La extensión compleja de estas funciones es,

$$
\operatorname{sen} z=\frac{\mathbb{e}^{i z}-\mathbb{e}^{-i z}}{2 i} \text { y } \cos z=\frac{\mathbb{e}^{i z}+\mathbb{e}^{-i z}}{2}, \text { para todo } z \in \mathbb{C}
$$

Usando $\log z=\ln |z|+i \arg (z), \quad z \neq 0$; las funciones trigonométricas inversas se obtienen como 

a) $\operatorname{arcsen}(z)=\frac{1}{i} \log \left(i z+\left(1-z^{2}\right)^{1 / 2}\right)$
b) $\arccos (z)=\frac{1}{i} \log \left(z+\left(z^{2}-1\right)^{1 / 2}\right)$
c) $\arctan (z)=\frac{1}{2 i} \log \frac{z+i}{z-i}=-\frac{i}{2} \log \frac{z+i}{z-i}$.

\subsection{El algoritmo de Johan Bernoulli (1703)}

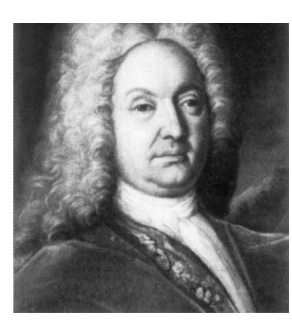

El problema de calcular primitivas de funciones racionales es viejo, tanto como el problema de calcular la derivada de una función. Según Ostrogradsky ([18, 1845]) Newton prefería desarrollar estas funciones en serie e integrar término a término (para evitar funciones trascendentes). Leibniz calculaba primitivas de funciones racionales obteniendo una factorización irreducible del denominador sobre los reales, y luego integrando la descomposición en fracciones parciales. Esto no incluía el manejo de todos los casos en los cuales los denominadores son cuadráticos. En el siglo XVIII Johan Bernoulli (1703) perfeccionó el método de descomposición en fracciones parciales y completó el método de Leibniz ([20, pág 353]). La prueba definitiva del algoritmo solo se pudo completar hasta que la prueba rigurosa del teorema fundamental del cálculo fue establecida por Gauss y Weiertrass. Este parece ser el algoritmo más viejo que se ha registrado sobre este tema. Es el método que se enseña hoy en los textos de cálculo. El problema principal del método es, por supuesto, la factorización completa del denominador. Ostrogradsky ([18]) presentó en 1845 un nuevo algoritmo que calcula la parte racional de la integral sin factorizar. Sin embargo este método solo se conocía en Rusia. En 1872 Hermite ([19]) publicó un algoritmo diferente para calcular la parte racional de la integral también sin factorizar (ver sección 1.12).

El algoritmo de Bernoulli (1703). Este método no es computacionalmente eficiente debido al costo de factorizar en $\mathbb{R}[x]$ pero tiene importancia teórica.

Supongamos que $f=P / Q$ con $P, Q \in \mathbb{R}[x]$. Después de dividir los polinomios (división con resto), podemos suponer que $f=C+A / Q$ con $C, A, Q \in \mathbb{R}[x], \operatorname{mcd}(A, Q)=1$ y grado $(A)<\operatorname{grado}(Q)$. Se factoriza $Q$ como

$$
Q=\prod_{i=1}^{n}\left(x-\alpha_{i}\right)^{n_{i}} \prod_{j=1}^{m}\left(x^{2}+b_{j} x+c_{j}\right)^{m_{j}}
$$

con las funciones cuadráticas irreducibles. La descomposición parcial de $f$ es

$$
f=S+\sum_{i=1}^{n} \sum_{k=1}^{n_{i}} \frac{A_{i k}}{\left(x-\alpha_{i}\right)^{k}}+\sum_{j=1}^{m} \sum_{k=1}^{m_{j}} \frac{B_{j k} x+C_{j k}}{\left(x^{2}+b_{j} x+c_{j}\right)^{k}}
$$

El algoritmo para calcular la integral es ([17, pág,36]), 
a) Calcular $\int C$ (no ofrece problema, pues $C$ es un polinomio).

b) Calcular cada integral $\int \frac{A_{i k}}{\left(x-\alpha_{i}\right)^{k}}=\left\{\begin{array}{lll}\frac{A_{i k}\left(x-\alpha_{i}\right)^{1-k}}{(1-k)} & \text { si } \quad k>1, \\ A_{i 1} \log \left(x-\alpha_{i}\right) & \text { si } \quad k=1\end{array}\right.$

c) Calcular las integrales con los denominadores cuadráticos. Recordemos que $b_{j}^{2}-4 c_{j}<0$. Sea $\Delta_{j}=4 c_{j}-b_{j}^{2}>0$. Hay dos casos.

- Si $k=1$,

$$
\int \frac{B_{j 1} x+C_{j 1}}{\left(x^{2}+b_{j} x+c_{j}\right)}=\frac{B_{j 1}}{2} \log \left(x^{2}+b_{j} x+c_{j}\right)+\frac{2 C_{j 1}-b_{j} B_{j 1}}{\sqrt{\Delta_{j}}} \arctan \left(\frac{2 x+b_{j}}{\sqrt{\Delta_{j}}}\right)
$$

- Si $k>1$, se usa la siguiente fórmula recursiva, hasta que $k-1=1$, y se integra como el caso anterior,

$$
\int \frac{B_{j 1} x+C_{j 1}}{\left(x^{2}+b_{j} x+c_{j}\right)^{k}}=\frac{\left(2 C_{j k}-b_{j} B_{j k}\right) x+b_{j} C_{j k}-2 C_{j} B_{j k}}{(k-1) \Delta_{j}\left(x^{2}+b_{j} x+c_{j}\right)^{k-1}}+\int \frac{(2 k-3)\left(2 C_{j k}-b_{j} B_{j k}\right)}{(k-1) \Delta_{j}\left(x^{2}+b_{j} x+c_{j}\right)^{k-1}}
$$

¿Descomposición en fracciones parciales?. La descomposición en fracciones parciales se usa en integración de funciones racionales y en el cálculo de la inversa de la transformada de Laplace. Pero "una descomposición en fracciones parciales" depende de la factorización que se tenga. Hay varios métodos de descomposición en fracciones parciales basados por ejemplo en la descomposición "libre de cuadrados del denominador" (ver [17, Sección 2.8 ],[25]).

Casos simples. Para polinomios que factorizan de manera simple, hay varias técnicas rápidas y muy eficientes para obtener los coeficientes de la descomposición en el estilo del algoritmo de Bernoulli (ver [21]). Por ejemplo,

a) $\frac{1}{[D(x)+a][D(x)+b]}=\frac{1}{b-a}\left(\frac{1}{D(x)+a}-\frac{1}{D(x)+b}\right), a \neq b$

b) Sea $Q(x)=x^{2}+b x+c$ con $Q(a) \neq 0$. Entonces $\frac{1}{(x-a)\left(x^{2}+b x+c\right)}=\frac{1}{Q(a)}\left(\frac{1}{x-a}-\frac{x+a+b}{x^{2}+b x+c}\right)$

c) $\operatorname{Si} P(x) / Q(x)$ es una fracción propia con la cuadrática $Q(x)=a x^{2}+b x+c$ irreducible, entonces

$$
\frac{P(x)}{\left(a x^{2}+b x+c\right)^{n}}=\frac{A_{1} Q^{\prime}(x)+B_{1}}{Q(x)}+\frac{A_{2} Q^{\prime}(x)+B_{2}}{Q^{2}(x)}+\ldots+\frac{A_{n} Q^{\prime}(x)+B_{n}}{Q^{n}(x)}
$$


d) Todas las raíces simples: Cuando $Q(x)$ tiene $n$ raíces simples $\alpha_{1}, \alpha_{2}, \cdots, \alpha_{n}$ y grado $P<$ grado $Q$, se puede usar los residuos en la expansión de Laurent de la función racional $P / Q$ en los polos $\alpha_{i}$ :

$$
\frac{P(x)}{Q(x)}=\sum_{k=1}^{n} \frac{c_{k}}{x-\alpha_{k}} \text { con } c_{k}=\frac{P\left(\alpha_{k}\right)}{Q^{\prime}\left(\alpha_{k}\right)}
$$

Este método se conoce como "método de los residuos" (por su origen el cálculo de residuos en funciones de variable compleja) y se puede generalizar a raíces de multiplicidad $m>1$ (ver [17, pág 54]). Si $\operatorname{mcd}\left(Q, Q^{\prime}\right)=1$ entonces todas las raíces de $Q$ son simples ${ }^{1}$.

\section{Ejemplo 1.4}

Calcular $\int \frac{1}{-3+x+x^{2}+x^{3}}$

$$
\begin{aligned}
\int \frac{1}{-3+x+x^{2}+x^{3}} & =\int \frac{1}{(x-1)\left(x^{2}+2 x+3\right)} \\
& \left.=\int \frac{1}{6}\left(\frac{1}{x-1}-\frac{x+3}{\left(x^{2}+2 x+3\right)}\right), \text { usando la fórmula b }\right) \\
& =\frac{1}{6}\left(\log (x-1)-\frac{1}{2} \log \left(x^{2}+2 x+3\right)-\frac{4}{\sqrt{8}} \arctan \left(\frac{2 x+2}{\sqrt{8}}\right)\right)
\end{aligned}
$$

(se usó la fórmula 1.1, con $\Delta_{1}=8$ )

\section{Ejemplo 1.5}

Calcular $\int \frac{x+1}{-15-13 x+3 x^{2}+x^{3}}$.

\section{(* Seguimos el algoritmo usando Mathematica *)}

Clear $[P, Q, Q p]$

$P\left[X_{-}\right]=x+1 ;$

$Q=-15-13 x+3 x^{\wedge} 2+x^{\wedge} 3$;

(* Derivada de $\mathbf{Q} *$ )

${ }^{1}$ Si $Q$ tiene una raíz $\alpha$ de multiplicidad $m>1$ entonces $Q=(x-\alpha)^{m} R(x)$. Por lo tanto $Q^{\prime}=m(x-\alpha)^{m-1} R(x)+(x-\alpha)^{m} R(x)$ con lo cual $\operatorname{mcd}\left(Q, Q^{\prime}\right) \neq 1$. 


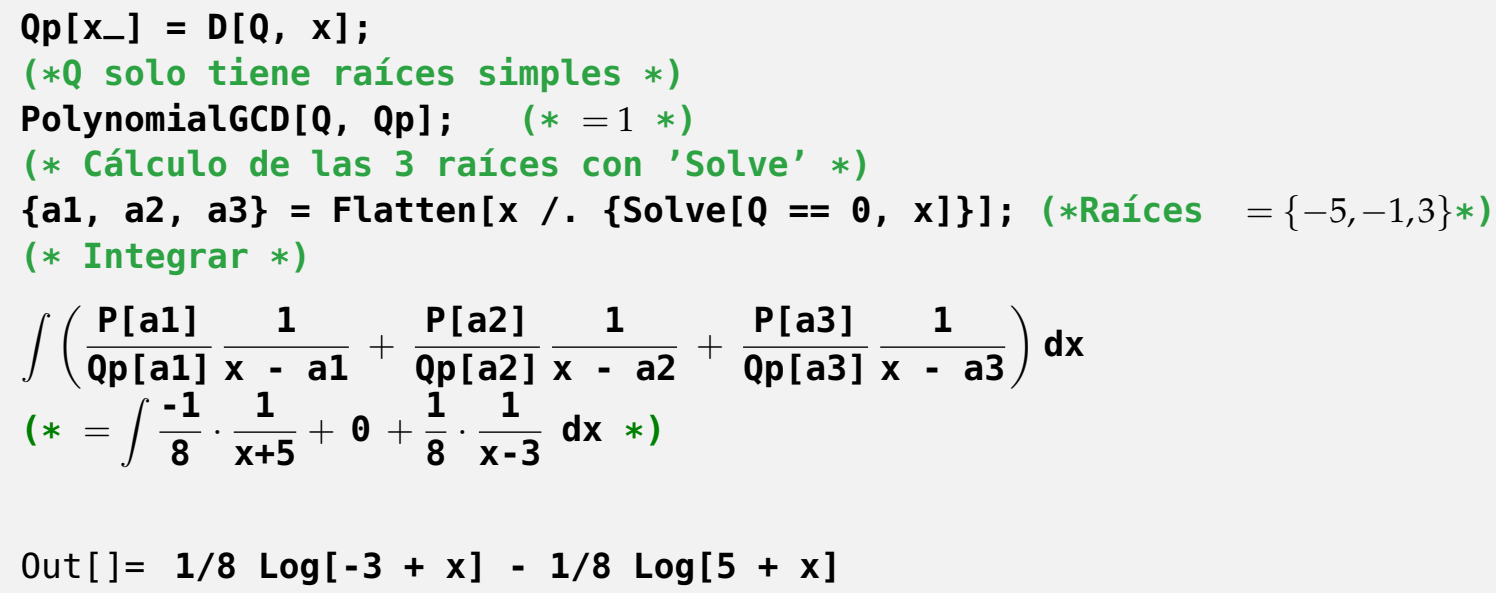

Caso de polinomios irreducibles en $\mathbb{Q}[x]$. Hay muchos polinomios de apariencia sencilla que son irreducibles en $\mathbb{Q}[x]$ o cuya factorización en $\mathbb{Q}[x]$ no se adapta al algoritmo de Bernoulli. Algunos ejemplos los podemos encontrar en [22]. Por ejemplo,
a) $x^{5}+x+1=\left(x^{2}+x+1\right)\left(x^{3}-x^{2}+1\right)$
b) $x^{3}-x^{2}+1$
c) $x^{7}+x+1$
d) $x^{6}-5 x^{4}-8 x^{3}-2 x^{2}+2 x+1$

No todo está perdido. Como veremos más adelante, integrar funciones racionales no requiere siempre obtener la factorización del denominador en el estilo del algoritmo de Bernoulli. Hay reducciones que no ocupan factorizar y simplifican, muchas veces, el cálculo de las primitivas.

\section{Ejemplo 1.6} Consideremos la integral racional $\int \frac{1}{x^{7}+x+1}$. Al hacer el cálculo con MATHEMATICA obten-
emos

$$
\begin{aligned}
& \operatorname{In}[]:=\int \frac{1}{x^{7}+x+1} d x \\
& \text { Out }[]=\operatorname{RootSum}\left[1+\# 1+\# 1^{7} \&, \log [x-\# 1] /\left(1+7 \# 1^{6}\right) \&\right]
\end{aligned}
$$

Este $Q=x^{7}+x+1$ no factoriza en $\mathbb{Q}[x]$ y tampoco las raíces se pueden expresar en términos de radicales en $\mathbb{R}$. Pero sus raíces son simples pues $\operatorname{mcd}\left(Q, Q^{\prime}\right)=1$; así que podemos dar una respuesta simbólica. 
Si las raíces de $Q$ son $\alpha_{1}, \alpha_{2}, \cdots, \alpha_{7}$, entonces, aplicando el método de los residuos,

$$
\begin{aligned}
\int \frac{1}{x^{7}+x+1} d x & =\int \sum_{k=1}^{7} \frac{1}{Q^{\prime}\left(\alpha_{k}\right)} \cdot \frac{1}{x-\alpha_{k}} d x \\
& =\sum_{k=1}^{7} \frac{\log \left(x-\alpha_{k}\right)}{7 \alpha_{k}^{6}+1}+C
\end{aligned}
$$

Esta es la interpretación de la salida "extraña" que nos da MAтHEMATica. Esta fórmula es útil para aproximar integrales definidas una vez calculadas la raíces aproximadas ([5]).

En la práctica computacional, no se factoriza el denominador totalmente para obtener la descomposición en factores parciales, en vez de eso se usa una factorización "libre de cuadrados". En todo caso no siempre es posible factorizar: Como se sabe, para polinomios de grado mayor o igual a cinco no siempre es posible encontrar las raíces del polinomio en términos de radicales.

Aunque $Q(x)=x^{6}-5 x^{4}-8 x^{3}-2 x^{2}+2 x+1$ no factoriza en $\mathbb{Q}[x]$, se puede hacer reducciones que solo ocupan operaciones con polinomios. En el capítulo sobre "integración de funciones racionales" (sección 1.11) vamos a ver como se calcula la integral $\int \frac{6 x^{5}+6 x^{4}-8 x^{3}-18 x^{2}+8 x+8}{x^{6}-5 x^{4}-8 x^{3}-2 x^{2}+2 x+1}$. Por ahora, veamos en el siguiente ejemplo, como va a quedar el resultado.

\section{Ejemplo 1.7}

Vamos a calcular $\int \frac{6 x^{5}+6 x^{4}-8 x^{3}-18 x^{2}+8 x+8}{x^{6}-5 x^{4}-8 x^{3}-2 x^{2}+2 x+1}$ con el método de Hermite y el método de Rothstein/Trager:

El cálculo solo requiere operaciones con polinomios y calcular las raíces del polinomio $R(z)=\left(-2-2 z+z^{2}\right)^{3}$ (ver sección 1.11).

$$
\begin{aligned}
\int \frac{6 x^{5}+6 x^{4}-8 x^{3}-18 x^{2}+8 x+8}{x^{6}-5 x^{4}-8 x^{3}-2 x^{2}+2 x+1} & =(1+\sqrt{3}) \log \left[1+x+\sqrt{3} x+\sqrt{3} x^{2}-x^{3}\right] \\
& +(1-\sqrt{3}) \log \left[-1-x+\sqrt{3} x+\sqrt{3} x^{2}+x^{3}\right]
\end{aligned}
$$




\subsection{Principio de Laplace, 1812.}

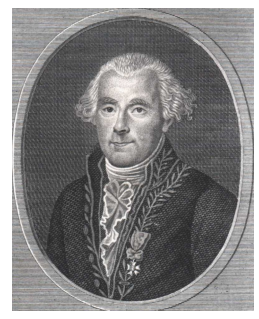

Laplace, en el primer libro de la "Théorie analytique des probabilités" [7, pág 5], estableció en 1812 por primera vez una teoría consistente de funciones "elementales" y la integración en términos finitos, en forma de esbozo general. Por ejemplo, Laplace indica que con su razonamiento, que la integral $\int \frac{d x}{\sqrt{1+\alpha x^{2}+\beta x^{4}}}$ no tienen primitiva elemental, sin embargo Liouvlle señala que "la demostración no se ha publicado y nosotros ignoramos de qué naturaleza esta demostración podría ser" ([23, pág 145]).

En todo caso, Liouville reconoce que este esbozo sobre el tema de integración en términos finitos es notable porque lo cita casi completo en su "Premier Memoire sur la détermination des intégrales dont la valeur est algébrique" ([23, pág 144]).

La cita de Lioville que aquí presentamos, en traducción libre, sigue la versión en inglés de Luetzen ([20, págs 357-58]): Habiendo introducido las funciones algebraicas, exponencial y logarítmicas, Laplace continúa:

Estas cantidades son esencialmente distintas: la exponencial $a^{x}$, por ejemplo, nunca puede ser idéntica a una función algebraica de $x$. De hecho, cada función algebraica es reducible a una serie descendente de la forma $k x^{m}+k^{\prime} x^{n-n^{\prime}}+\ldots$; pero es fácil demostrar que cuando $a$ es mayor que la unidad y $x$ es infinita, $a^{x}$ es infinitamente mayor que $k x^{n}$, por muy grande que $k$ y $n$ puedan ser. Del mismo modo, es fácil ver que cuando $x$ es infinito, $x$ es infinitamente mayor que $k(\log x)^{n}$.

Por lo tanto, las funciones exponenciales, algebraicas y logarítmicas no pueden reducirse de una a la otra; las cantidades algebraicas están en el medio entre las exponenciales y los logaritmos, de hecho, cuando la variable es infinita, podemos considerar los exponentes como infinitos en las exponenciales, finito en las algebraicas e infinitamente pequeñas en las cantidades logarítmicas.

Además, uno puede en principio establecer que una función radical de una variable no puede ser idéntica a una función racional de la misma variable o con otra función radical. Por lo tanto, $\left(1+x^{3}\right)^{1 / 4}$ es esencialmente diferente de $\left(1+x^{3}\right)^{1 / 3}$ y de $(1+x)^{1 / 2}$. Estos principios, que se basan en la naturaleza de las funciones, pueden ser de gran utilidad en la investigación analítica, ya que nos indican la forma que necesariamente deben tener este tipo de funciones que andamos buscando y mostrar que esta forma no existe en un gran número de casos. Sin embargo, uno debe entonces estar absolutamente seguro de no omitir ninguna de las formas posibles. Por lo tanto, puesto que la diferenciación permite a la exponencial y las cantidades radicales subsistir y sólo hace que las cantidades logarítmicas desaparezcan cuando están multiplicadas por las constantes, se puede concluir que la integral de una función diferencial no puede incluir ninguna otra exponencial y ningún radical que los que ya figuran en el presente función. De este modo, me he dado cuenta que no se puede obtener la integral $\int \frac{d x}{\sqrt{1+\alpha x^{2}+\beta x^{4}}}$ como una función finita explícita o implícita. Del mismo modo, he demostrado que las ecuaciones diferenciales en derivadas parciales lineales de segundo orden y de tres variables, no pueden ser integradas de forma finita, y esto me ha llevado a un método general de integrar éstas en esta forma cuando sea posible. En los otros casos, no se puede obtener una integral finita sino por medio de integrales definidas. [Laplace 1812, pp 4-5]' 
Más adelante va a ir quedando claro como la "forma" del integrando y de la primitiva es crucial en la decisión de si una función tiene o no primitiva elemental.

Acerca de esto, en esta primera memoria de Liouville sobre el tema ([23]), él inicia probando que la integral $\int \frac{d x}{\sqrt{1+x^{4}}}$ no tiene primitiva elemental. Luetzen ([20, págs 370-372]) indica que el camino que Liouville siguió (de acuerdo a su manuscritos) fue el de expresar esta integral en "la forma"

$$
\int \frac{d x}{\sqrt{1+x^{4}}}=\frac{M+P \sqrt{1+x^{4}}}{Q}
$$

con $M, P$ y $Q$ polinomios en $\mathbb{Z}[x]$. La justificación elude a los principios de Laplace: La primitiva solo podría tener el mismo tipo de radicales que aparece en el integrando (no cúbicas, ni de otro orden, ni exponenciales ni logaritmos, etc.). Luego prueba que es imposible encontrar $M, P$ y $Q$ que satisfagan esta identidad.

\subsection{Teorema de P. Chebyshov, 1853.}

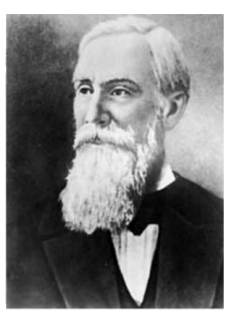

Nuestro interés está ahora en un teorema de P. Chebyshov porque, aunque es posterior (y complementaria) de la teoría establecida por Liouville, es un teorema de fácil aplicación. P. Chebyshov estaba interesado en casos especiales de funciones algebraicas y su trabajo sobre la integración de este tipo de funciones está conectado de manera muy cercana con el trabajo de Abel, Liouville y Ostrogradsky. En el artículo "Sur l'integration des différentielles irrationelles" (1853, [3]) P. Chebyshov resuelve el problema de obtener la 'parte logarítmica' de la integral

$$
\int \frac{P(x)}{Q(x)} \frac{d x}{\sqrt[m]{R(x)}}
$$

donde $P, Q$ y $R$ son polinomios y $m$ es un entero positivo. Pero el artículo es más bien conocido por la solución completa del problema de la integración del 'binomio diferencial'

$$
\int x^{m}\left(a+b x^{n}\right)^{p} d x
$$

Ya Goldbach y Euler habían mostrado que este tipo de integral es expresable en términos de funciones elementales en los casos en que $p$ es un entero, $(m+1) / n$ es un entero o $(m+1) / n+p$ es un entero. P. Chebyshov demostró, con métodos analíticos, que estos son los únicos casos en los que esta integral se puede expresar en términos de funciones elementales.

¿Se puede saber si una función tiene primitiva elemental? . Walter Mora F.

Derechos Reservados @ 2015 Revista digital Matemática, Educación e Internet (http://tecdigital.tec.ac.cr/revistamatematica/) 


\section{Sur l'intégration des diflérentielles irrationnelles.}

\section{\$ I.}

Si la différentielle $\frac{f_{0} x}{F_{0} x} \frac{d x}{\sqrt[m]{6 x}}$, composée d'une fraction rationuelle $\frac{f_{0} x}{F_{0} x}$ et d'une racine d'une fonction entière $0 x$, s'intègre à l'aide des signes a]gébriques et logarithmiques, nous savons, d'après les recherches ingénieuses d'Abel et de M. Liouville, que l'intégrale $\int \frac{f_{0} x}{F_{0}^{x} x} \frac{d x}{\sqrt[m]{\theta x}}$ se présentera sous la forme suivante:

$$
U+A^{0} \log V^{0}+A^{\prime} \log V^{\prime}+A^{\prime \prime} \log V^{\prime \prime}+\ldots,
$$

où $U, V^{0}, \nabla^{\prime}, V^{\prime \prime}, \ldots$, sont des fonctions rationnelles de $x$ et $\sqrt[m]{O x}$; $A^{0}, A^{\prime}, A^{\prime \prime}, \ldots$, sont des quantités constantes.

Figura 1.1: P.L. Tchebychef. "Sur l'integration des différentielles irrationelles" (en Oeuvres, 1899), [3]

Para la presentación de este teorema definitivamente lo mejor es seguir la exposición del libro "Cálculo diferencial e Integral" de N. Piskunov [10, págs. 375-378].

\section{Teorema 1.1 (Chebyshov, 1853).}

Si $m, n$ y $p$ son números racionales, entonces la integral

$$
\int x^{m}\left(a+b x^{n}\right)^{p} d x
$$

se puede expresar en términos de funciones elementales si y sólo si al menos uno de los números $p, \frac{m+1}{n}$ o $\frac{m+1}{n}+p$, es entero.

En el caso de que la integral se puede expresar en términos de funciones elementales, la manera de obtener la primitiva es vía sustituciones [10, págs. 375-378]. Estas sustituciones convierten la integral en una función racional que, por el algoritmo de J. Bernoulli, siempre tiene primitiva elemental. La otra parte de la prueba del teorema de Chebyshov no es elemental, se puede ver en [8, págs. 37-39] y en español en [9, págs. 45-48].

Aplicación. El teorema se puede usar para decidir si una función de la forma $x^{m}\left(a+b x^{n}\right)^{p}($ "binomio diferencial") tiene primitiva elemental y, también para el cálculo.

Para calcular, primero se hace la sustitución $x=u^{1 / n}$, entonces $d x=\frac{1}{n} u^{\frac{1}{n}-1} d u$ y

$$
\int x^{m}\left(a+b x^{n}\right)^{p} d x=\frac{1}{n} \int u^{q}(a+b u)^{p} d u \text { con } q=\frac{m+1}{n}-1
$$


a) Caso I: Si $p$ es entero y $q$ es racional. Si $q=\frac{h}{d}$, se hace la sustitución $u=t^{d}$.

b) Caso II: Si $\frac{m+1}{n}$ es entero y $p$ es racional. $\operatorname{Si} p=\frac{h}{d}$, se hace la sustitución $a+b u=t^{d}$.

c) Caso III. Si $\frac{m+1}{n}+p$ es entero y $p$ es racional. Primero se transforma la integral,

$$
\frac{1}{n} \int u^{q}(a+b u)^{p} d u=\frac{1}{n} \int u^{q+p}\left(\frac{a+b u}{u}\right)^{p} d u \text { con } q=\frac{m+1}{n}-1
$$

Si $q=\frac{h}{d}$, se hace la sustitución $\frac{a+b u}{u}=t^{d}$.

\section{Ejemplo 1.8}

Consideremos la integral $\int \frac{d x}{\sqrt[3]{x} \sqrt{x+1}}=\int x^{-1 / 3}(1+x)^{-1 / 2} d x$. En este caso $m=-\frac{1}{3}$, $\frac{m+1}{n}=\frac{2}{3}$ y $\frac{m+1}{n}+p=\frac{2}{3}-\frac{1}{2}$. Como ninguno de estos números es entero, la integral no se puede expresar en términos de funciones elementales.

\section{Ejemplo 1.9}

Consideremos la integral $\int \frac{d x}{\sqrt{\left(4 x-x^{2}\right)^{3}}}=\int x^{-3 / 2}(4-x)^{-3 / 2} d x$. Como $\frac{m+1}{n}+p=-\frac{1}{2}-$ $\frac{3}{2}=-2$, la integral sí se puede expresar en términos de funciones elementales. Usando las indicaciones de sustitución mencionadas, se obtiene

$$
\int x^{-1 / 3}(1+x)^{-1 / 2} d x=\frac{1}{4} \frac{x-2}{\sqrt{x(4-x)}}+K
$$

\section{Ejemplo 1.10}

a) $\int \sqrt{\operatorname{sen} x} d x$ no es elemental: Si $u=\operatorname{sen} x$ obtenemos 


$$
\int \sqrt{\operatorname{sen} x} d x=\int u^{1 / 2}\left(1-u^{2}\right)^{1 / 2} d u
$$

que, de acuerdo al teorema de Chebyshov, no se puede expresar en términos de funciones elementales.

b) $\int \sqrt{\tan x} d x$ es elemental. Si $u=\sqrt{\tan x}, u^{2}=\tan x$, la integral queda

$$
\int \sqrt{\tan x} d x=\int 2 u^{2}\left(u^{4}+1\right)^{-1} d u
$$

que si pasa el test de Chebyshov pues $p=-1$.

c) Consideremos $\int\left(1-x^{n}\right)^{1 / k} d x$. Como $m=0$ y $p=\frac{1}{k}$, entonces la integral es elemental solo si $\frac{1}{n}$ es entero o $1 / k$ es entero o $\frac{1}{n}+\frac{1}{k}$ es entero, es decir, solo si $k= \pm 1$, o $n= \pm 1$ o $k=n=2$ o $n=-k$ (en otro caso $\left.\left|\frac{1}{n}+\frac{1}{k}\right|<1\right)$.

Si $k>0$, esto solo nos deja $1-x^{n}, \sqrt[k]{1-x}, \sqrt[k]{1-1 / x}, \sqrt{1-x^{2}}$ y $\sqrt[k]{1-x^{-k}}$ con primitiva elemental.

Longitud de arco. La longitud (de arco) de una curva suave de ecuación $y=f(x)$ desde $x=a$ hasta $x=b$, se calcula con la fórmula

$$
s=\int_{a}^{b} \sqrt{1+\left(f^{\prime}(x)\right)^{2}} d x
$$

Como se ve, son pocas las integrales de este tipo que se pueden calcular usando el teorema fundamental del cálculo por la escasez de primitivas elementales.

Por ejemplo, si $f(x)=\frac{1}{x}$ entonces, $s=\int \sqrt{1+x^{-4}} d x$ no es elemental.

\subsection{Primitivas elementales y funciones inversas}

Usando integración por partes, la integral de una función $f$ se puede expresar en términos de la integral de su inversa $f^{-1}$. Además de ser un recurso de cálculo, también se puede usar como método de decisión en el caso de que se conozca que $f$ o $f^{-1}$ tiene o no tiene primitiva elemental.

Si $f$ es invertible en $[a, b]$ y si $f$ y $f^{-1}$ tienen derivada continua, entonces aplicando integración por partes con $u=f(x)$ y $d v=d x$, se obtiene 


$$
\begin{aligned}
\int f(x) d x & =x f(x)-\int x f^{\prime}(x) d x \\
& =x f(x)-\int f^{-1}(f(x)) f^{\prime}(x) d x
\end{aligned}
$$

Aplicando la sustitución $t=f(x)$ en la integral de la derecha, obtenemos la primitiva $G(t)=\int f^{-1}(t) d t$. Sustituyendo queda

$$
\int f(x) d x=x f(x)-G(f(x))
$$

A veces, abusando del lenguaje, se escribe

$$
" \int f(x) d x=x f(x)-\int^{f(x)} f^{-1}(y) d y "
$$

\section{Ejemplo 1.11}

a) $\int \ln x d x$ es elemental pues $\int^{\ln x} e^{y} d y$ es elemental:

$$
\int \ln x d x=x \ln x-\int^{\ln x} e^{y} d y
$$

b) $\int \sqrt{\ln x} d x=x \sqrt{\ln x}-\int^{\ln x} e^{y^{2}} d y$

Así, $\int \sqrt{\ln x} d x$ no es elemental porque (como veremos más adelante) $\int e^{x^{2}} d x$ no es elemental.

c) $\int \frac{1}{\ln x} d x=\frac{x}{\ln x}-\int^{1 / \ln x} e^{1 / y} d y$

Así, $\int e^{1 / y} d x$ no es elemental porque (como veremos más adelante) $\int \frac{1}{\ln x} d x$ no es elemental. 


\subsection{El principio de Liouville}

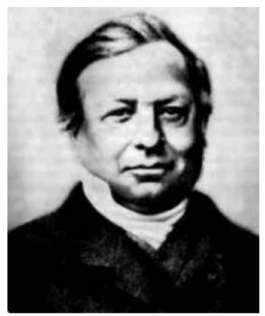

El estudio sistemático del problema de decidir cuando una integral tiene primitiva elemental inicio en el siglo XIX. Una década después de Laplace, J. Liouville establece y prueba un teorema más general y preciso: Hablando informalmente, Liouville establece que si la primitiva de una función elemental $f$ es una función elemental, entonces esta primitiva se puede expresar usando solo las funciones que aparecen en el integrando y una combinación lineal de logaritmos de tales funciones. El teorema de Liouville nos da un criterio de integración de funciones elementales en términos de la solución de una ecuación diferencial de primer orden con una función racional.

\section{Ejemplo 1.12}

a) $\int \frac{1}{2} \mathbb{e}^{i x}+\frac{1}{2} \mathbb{e}^{-i x} d x=-\frac{1}{2} i \mathbb{e}^{i x}+\frac{1}{2} \mathbb{e}^{-i x}+K$

b) $\int \frac{1}{\sqrt{1-x^{2}}} d x=-i \log \left(\sqrt{1-x^{2}}+i x\right)+K$

c) $\int \log \left(x+\sqrt{x^{2}-1}\right) d x=x \log \left(x+\sqrt{x^{2}+-1}\right)-\sqrt{x^{2}-1}+K$

Para enunciar el teorema "fuerte" de Liouville necesitamos ubicarnos con las funciones algebraicas y trascendentes.

Funciones algebraicas. Recordemos que una función algebraica (ver por ejemplo [13]) $y=y(x)$ es una función que satisface una ecuación de la forma

$$
R_{n}(x) y^{n}+R_{n-1}(x) y^{n-1}+\cdots+R_{0}(x)=0, \quad \text { con } R_{n}(x) \not \equiv 0 .
$$

donde cada $R_{i}(x)$ una función racional. Multiplicando a ambos lados por el máximo común denominador de los denominadores nos queda

$$
P(x, y)=a_{n}(x) y^{n}+a_{n-1}(x) y^{n-1}+\cdots+a_{0}(x)=0, \quad \text { con } a_{n}(x) \not \equiv 0 .
$$

donde cada $a_{i}(x)$ es un polinomio con coeficientes en algún conjunto. Desde el punto de vista computacional interesa que $a_{i}(x) \in \mathbb{Q}[x]$. 
Las funciones racionales son algebraicas: Si $y=\frac{P(x)}{Q(x)}$ entonces $y$ es algebraica porque satisface la ecuación

$$
Q(x) y-P(x)=0 \text {. }
$$

Una expresión que puede ser formada a partir de $x$ y ciertas constantes aplicando un número finito de veces las operaciones de suma, resta, producto, división y 'extracción de raíces', se llama una función algebraica explícita. Por ejemplo,

$$
y=a-\frac{b}{x^{2}}+\sqrt[3]{\frac{a+x^{4}}{a+\sqrt{b+d x^{3}}}}+\sqrt{\frac{1}{1+x^{3}}+\sqrt{a+b x}}
$$

puede ser obtenida a partir de $x, a, b, d$ aplicando las operaciones indicadas.

Se sabe que las funciones algebraicas explícitas satisfacen la definición de funciones algebraicas, pero como las ecuaciones polinomiales de grado $>4$ no pueden ser, en general, resueltas por radicales, no todas las funciones algebraicas puede ser explícitas.

La suma, resta, producto, división y "extracción de raíces" de funciones algebraicas vuelve a ser una función algebraica. Si y es algebraica y es invertible, su inversa es algebraica. Además las funciones algebraicas son continuas en su dominio y tienen un número finito de ceros.

Funciones trascendentes. Las funciones que no son algebraicas se llaman trascendentes. Por ejemplo, $\operatorname{sen} x, \cos x, \tan x, \ln x$ y sus inversas son trascendentes. Veamos un par de pruebas.

sen $x$ es trascendente: Si fuera algebraica, tendría un número finito de ceros, pero $\operatorname{sen}(k \pi)=0$ para todo $k \in \mathbb{Z}$. También se puede probar que sen $x$ es trascendente usando el hecho de que $P(x, \operatorname{sen} x)$ es una función continua (ver apéndice ??).

$y=\ln x$ es trascendente (por tanto también su inversa $y=e^{x}$ ). La prueba de este hecho usa una idea que vamos a encontrar varias veces. La idea de la prueba es esta: $\operatorname{Si} \ln x$ satisface la ecuación polinomial, $\ln x$ debe ser racional y eso no puede pasar. Iniciamos probando que $\ln x$ no puede ser una función racional.

Si $\ln x=\frac{P(x)}{Q(x)}$ con $P$ y $Q$ coprimos, entonces $\frac{1}{x}=\frac{Q P^{\prime}-P Q^{\prime}}{Q^{2}}$, es decir,

$$
Q^{2}=x\left(Q P^{\prime}-P Q^{\prime}\right)
$$

Por tanto $x$ es un factor de $Q$. Sea $Q(x)=x^{m} R(x)$, con $R(0) \neq 0$ y $m \geq 1$. Sustituyendo y dividiendo por $x^{m}$ obtenemos,

$$
\begin{aligned}
x^{m} R^{2} & =x\left[x^{m} R P^{\prime}-P\left(m x^{m-1} R+x^{m} R^{\prime}\right)\right] \\
& =x R P^{\prime}-m P R-x P R^{\prime}
\end{aligned}
$$

Pasando a restar y sacando $x$ a factor se tiene, 


$$
m P(x) R(x)=x\left[R P^{\prime}-P R^{\prime}-x^{m-1} R^{2}\right]
$$

Entonces $x$ divide a $P R$, como no divide a $R$, entonces divide a $P$, lo que no puede ser pues $P$ y $Q$ son coprimos.

Ahora supongamos que $y=\ln x$ es algebraica y que

$$
a_{N}(x)(\ln x)^{N}+a_{N-1}(x)(\ln x)^{N-1}+\cdots+a_{0}(x)=0, \quad \text { con } a_{N}(x) \not \equiv 0,
$$

donde la ecuación se escogió con el menor grado $N$ posible. Entonces

$$
(\ln x)^{N}+\frac{a_{N-1}(x)}{a_{N}(x)}(\ln x)^{N-1}+\cdots+\frac{a_{0}(x)}{a_{N}(x)}=0, \quad \text { con } N \geq 2
$$

Derivando obtenemos,

$$
\frac{N}{x}(\ln x)^{N-1}+\left(\frac{a_{N-1}(x)}{a_{N}(x)}\right)^{\prime}(\ln x)^{N-1}+\cdots=0, \quad \text { con } N \geq 2
$$

que es un polinomio de grado menor que $N$, por lo que lo único que queda es que los coeficientes (polinomios) se deben anular para satisfacer la ecuación. En particular,

$$
\frac{N}{x}+\left(\frac{a_{N-1}(x)}{a_{N}(x)}\right)^{\prime}=0 .
$$

Pero integrando obtenemos que $\ln x=-\frac{a_{N-1}(x)}{N a_{N}(x)}$, es decir, $\ln x$ sería una función racional. Pero esto no puede ser como ya probamos.

El teorema fuerte de Liouville es enunciado aquí en términos de una función $f\left(x, y_{1}, \ldots, y_{n}\right)$ que se expresa en términos de funciones elementales $y_{i}(x)$ pero que requiere que las derivadas $y_{i}^{\prime}$ sean algebraicas.

Teorema 1.2 (Lioville, 1835).

Sea $f\left(x, y_{1}, y_{2}, \ldots, y_{n}\right)$ una función algebraica donde cada $y_{i}=y_{i}(x)$ y cada $\frac{d y_{i}}{d x}$ es función algebraica de $x, y_{1}, \ldots, y_{n}$. Entonces $\int f\left(x, y_{1}, y_{2}, \ldots, y_{n}\right) d x$ es elemental si y sólo si

$$
\int f\left(x, y_{1}, y_{2}, \ldots, y_{n}\right) d x=U_{0}+\sum_{j=1}^{n} C_{j} \ln \left(U_{j}\right)
$$

donde $U_{0}$ es función algebraica de $x$ y cada $C_{j}$ es constante y cada $U_{j \geq 1}$ es función algebraica de $x, y_{1}, \ldots, y_{n}$. 
- Si $f\left(x, y_{1}, y_{2}, \ldots, y_{n}\right)$ es una función racional y cada $\frac{d y_{i}}{d x}$ es función racional de las funciones $x, y_{1}, \ldots, y_{n}$, entonces $U_{0}$ es una función racional de $x$ y cada $U_{j}, j \geq 1$, deben ser función racional de $x, y_{1}, \ldots, y_{n}$.

La notación es un poco fuerte, pero es en realidad sencilla.

\section{Ejemplo 1.13}

Sea $y_{1}=\mathbb{e}^{x}, y_{2}=\operatorname{sen} x \quad$ y $y_{3}=\cos x$.

Sea $f\left(x, y_{1}, y_{2}, y_{3}\right)=2 x+\frac{y_{3}}{y_{2}}+\frac{2+2 y_{1}}{y_{1}+x}$. En este caso, $f$ es racional

Como $\frac{d y_{1}}{d x}=y_{1}, \quad \frac{d y_{2}}{d x}=y_{3}, \quad$ y $\frac{d y_{3}}{d x}=-y_{2}$, se cumple que $f\left(x, \mathbb{e}^{x}, \operatorname{sen} x, \cos x\right)$ es algebraica y que las derivadas $\frac{d y_{i}}{d x}$ son funciones algeraicas de $x, y_{1}, y_{2}, y_{3}$.

El teorema de Liouville dice que $\int f\left(x, y_{1}, y_{2}, y_{3}\right) d x$ es elemental si y sólo si

$$
\int 2 x+\frac{y_{3}}{y_{2}}+\frac{2+2 y_{1}}{y_{1}+x} d x=U_{0}+\sum_{j=1}^{4} C_{j} \ln \left(U_{j}\right)
$$

con $U_{0}=U_{0}(x)$ algebraica y las $C_{j}$ son constantes y las $U_{j \geq 1}$ son funciones algebraicas de $x, y_{1}, y_{2} y_{3}$.

En este caso la integral efectivamente es elemental y

$$
\int 2 x+\frac{\cos x}{\operatorname{sen} x}+\frac{2 e^{x}+2}{x+e^{x}} d x=x^{2}+2 \ln \left(x+e^{x}\right)+\ln (\operatorname{sen}(x))+K
$$

y como se ve, $U_{0}=x^{2}, U_{1}=x+y_{1}=x+e^{x}$ y $U_{2}=y_{2}=\operatorname{sen}(x)$.

El teorema de Liouville tiene valor teórico y práctico y ha sido la base de el trabajo desarrollado en la rama de integración "en términos finitos". Hay dos casos especiales del teorema que nos dan herramientas para decidir si ciertas funciones importantes tienen o no primitiva elemental. 


\subsection{Caso especial del teorema de Lioville, 1835.}

Consideremos la función elemental $f(x) \mathbb{e}^{x^{2}}$ con $f \in \mathbb{Q}(x)$. De acuerdo al teorema de Liouville, $\int f(x) \mathbb{e}^{x^{2}} d x$ es elemental si y sólo si

$$
\int f(x) \mathbb{e}^{x^{2}} d x=U_{0}+\sum_{j=1}^{n} C_{j} \ln \left(U_{j}\right)
$$

con $U_{0}(x)$ una función racional y cada $U_{j \geq 1}=U_{j}\left(x, \mathbb{e}^{x^{2}}\right)$.

Derivando a ambos lados en (1.3) obtenemos

$$
f(x) \mathbb{e}^{x^{2}}=U_{0}^{\prime}+\sum_{j=1}^{n} C_{j} \frac{U_{j}^{\prime}}{U_{j}}
$$

Si $U_{0}=P / Q$ se obtiene, con un poco de esfuerzo, una ecuación diferencial que se debe satisfacer: $f(x)=U_{0}^{\prime}+2 x U_{0}$. Esta ecuación diferencial es la clave para demostrar, eso sí de manera algebraica, que $f(x) \mathbb{e}^{x^{2}}$ tiene o no tiene primitiva elemental. Tratar de resolver la ecuación diferencial (vía factor integrante) no ayuda en nada.

En general,

\section{Teorema 1.3 (Caso especial - Liouville, 1835)}

Si $f$ y $g$ son funciones racionales y $g$ no es constante, entonces

$$
\int f(x) \mathbb{e}^{g(x)} d x \text { es elemental si y sólo si existe } R(x) \text { racional, tal que } f(x)=R^{\prime}(x)+R(x) g^{\prime}(x)
$$

Hay una parte fácil en la prueba del teorema. Una dirección del teorema dice que si existe una función racional $R$ tal que $f(x)=f(x)=R^{\prime}(x)+R(x) g^{\prime}(x)$, entonces

$$
\int f(x) \mathbb{e}^{g(x)} d x=R(x) \mathbb{e}^{g(x)} .
$$

En efecto, derivando obtenemos 


$$
\begin{aligned}
\frac{d}{d x} R(x) \mathbb{e}^{g(x)} & =R^{\prime}(x) \mathbb{e}^{g(x)}+\mathbb{e}^{g(x)} R(x) \\
& =\mathbb{e}^{g(x)}\left(R^{\prime}(x)+g^{\prime}(x) R(x)\right) \\
& =f(x) \mathbb{e}^{g(x)}
\end{aligned}
$$

La otra dirección de la prueba es la que no es elemental.

\section{Ejemplo 1.14}

$\int \mathbb{e}^{x^{2}} d x$ no tiene primitiva elemental (y por tanto $\int \sqrt{\ln x} d x$ tampoco, según la sección 1.7).

En efecto, de acuerdo al teorema 1.3, $\int 1 \cdot \mathbb{e}^{x^{2}} d x$ es elemental si y sólo si existe $R(x)$ racional tal que

$$
1=R^{\prime}(x)+R(x) 2 x
$$

Sea $R(x)=\frac{P(x)}{Q(x)}$ con $\operatorname{mcd}(P, Q)=1$. Entonces tenemos

$$
1=\frac{P^{\prime} Q-Q^{\prime} P}{Q^{2}}+2 x \frac{P}{Q}=\frac{P^{\prime} Q-Q^{\prime} P+2 x P Q}{Q^{2}}
$$

de donde

$$
Q^{2}=-Q^{\prime} P+P^{\prime} Q+2 x P Q
$$

Como $Q \mid\left(P^{\prime} Q+2 x P Q\right)$ entonces $Q \mid Q^{\prime} P$ y como $\operatorname{mcd}(Q, P)=1$ se concluye que $Q \mid Q^{\prime}$. Pero la derivada $Q^{\prime}$ tiene menor grado que $Q$, por lo tanto la única posibilidad es que $Q$ sea constante. Digamos $Q \equiv q$

Entonces 1.4 se puede reescribir como

$$
q=P^{\prime}+2 x P
$$

Pero esto es imposible pues $P^{\prime}+2 x P$ tiene grado $\geq 1$ mientras que la constante tiene grado cero. 


\section{Ejemplo 1.15}

$\int x^{2 n} \mathbb{e}^{a x^{2}} d x$, con $n \in \mathbb{N}^{*}$ y $a \neq 0$, no tiene primitiva elemental. La prueba sigue un argumento similar al del ejemplo anterior (en [14] puede ver todos los detalles).

\section{Ejemplo 1.16}

$\int \frac{e^{x}}{x} d x$ no tiene primitiva elemental.

En efecto, de acuerdo al teorema 1.3, $\int \frac{1}{x} \cdot \mathbb{e}^{x} d x$ es elemental si y sólo si existe $R(x)$ racional tal que

$$
\frac{1}{x}=R^{\prime}(x)+R(x)
$$

Sea $R(x)=\frac{P(x)}{Q(x)}$ con $\operatorname{mcd}(P, Q)=1$. Entonces tenemos

$$
\frac{1}{x}=\frac{P^{\prime} Q-Q^{\prime} P}{Q^{2}}+\frac{P}{Q}=\frac{P^{\prime} Q-Q^{\prime} P+P Q}{Q^{2}}
$$

de donde

$$
Q\left(Q-x P^{\prime}-x P\right)=-x Q^{\prime} P
$$

Si $Q$ tiene una raíz $\alpha \neq 0$ de multiplicidad $k \geq 1$ entonces $Q=(x-\alpha)^{k} S(x)$ con $S(\alpha) \neq 0$. Entonces, como $P$ no comparte raíces con $Q$, la ecuación 1.6 se puede reescribir como

$$
(x-\alpha)^{k+m} N(x)=(x-\alpha)^{k-1} D(x), \text { con } N(\alpha) \neq 0, D(\alpha) \neq 0 \text { y } m \geq 0 .
$$

Como las multiplicidades son distintas, $Q$ no tiene raíces no nulas. Por tanto, si $Q$ tiene una raíz, debería ser $\alpha=0$. Sea $Q=C x^{k}$ con $C$ constante y $k \geq 1$. En este caso la ecuación 1.6 se puede reescribir como

$$
(x-\alpha)^{k+m} N(x)=(x-\alpha)^{k-1} D(x), \text { con } N(\alpha) \neq 0, D(\alpha) \neq 0 \text { y } m \geq 0 .
$$

se puede reescribir como

$$
x^{k+m} N(x)=x^{k} D(x), \text { con } N(0) \neq 0, D(0) \neq 0 \text { y } m \geq 1 .
$$


De nuevo las multiplicidades no coinciden, por lo que $Q$ es constante, $Q \equiv q$. Sustituyendo en 1.6 nos queda

$$
q=x P^{\prime}+x P
$$

que es imposible pues el lado derecho tiene grado $\geq 1$.

Se puede probar que otras integrales no tienen primitiva elemental usando identidades, sustituciones o el método de integración por partes.

\section{Ejemplo 1.17}

a) $\int \frac{1}{\ln t} d t$ no tiene primitiva elemental pues $\int \frac{e^{x}}{x} d x=\int \frac{1}{\ln t} d t$ usando la sustitución $x=\ln t$.

b) $\int \mathbb{e}^{x} \ln x d x$ no tiene primitiva elemental pues, integrando por partes con $u=\mathbb{e}^{x} \mathrm{y}$ $d v=\frac{1}{x} d x$, se obtiene

$$
\int \frac{e^{x}}{x} d x=\mathbb{e}^{x} \ln x-\int \mathbb{e}^{x} \ln x d x
$$

\subsection{Caso especial Liouville-Hardy, 1905.}

El siguiente caso especial fue obtenido por G. Hardy ([6]) en 1905 y se aplica a funciones de la forma $f(x) \ln x$.

\section{Teorema 1.4 (Liouville-Hardy, 1905)}

Si $f(x)$ es una función racional, entonces $\int f(x) \ln x d x$ es elemental si y sólo si existe una función racional $R(x)$ y una constante $C$ tal que

$$
f(x)=\frac{C}{x}+R^{\prime}(x)
$$


La prueba de este teorema tiene una parte fácil: $\operatorname{Si} f(x)=\frac{C}{x}+R^{\prime}(x)$ entonces $\int f(x) \ln x d x$ es elemental. En efecto,

$$
\begin{aligned}
\int f(x) \ln x d x & =\int\left(\frac{C}{x}+R^{\prime}(x)\right) \ln x d x \\
& =\int \frac{C \ln x}{x} d x+\int \ln x R^{\prime}(x) d x \\
& =\frac{C \ln ^{2}(x)}{2}+\ln x R(x)-\int \frac{1}{x} R(x) d x, \text { integrando por partes con } u=\ln x \text { y } d v=R^{\prime}(x) d x
\end{aligned}
$$

$\int \frac{R(x)}{x} d x$ es elemental porque $\frac{R(x)}{x}$ racional. Por lo tanto $\int f(x) \ln x d x$ es elemental.

La otra parte de la prueba no es elemental.

\section{Ejemplo 1.18}

En el teorema 1.4, para probar que una integral es elemental, se debe encontrar dos cosas: una constante $C$ y una función racional $R$ tal queda

$$
R^{\prime}(x)=f(x)-\frac{C}{x}
$$

Integrando tenemos

$$
R=\int f(x) d x-C \ln x
$$

por lo tanto, si $C=0$ y $f(x)$ es un polinomio, el teorema se cumple.

$$
\int P(x) \ln x d x \text { es elemental si } P \text { es un polinomio. }
$$

Esto es algo fácil de verificar (y calcular) usando integración por partes.

\section{Ejemplo 1.19}

Sea $Q$ es un polinomio con todos sus ceros simples y no nulos, digamos $Q(x)=\left(x-\alpha_{1}\right) \cdots\left(x-\alpha_{n}\right)$, entonces $\int \frac{\ln x}{Q(x)} d x$ no es elemental. 
Esto es así pues sería $R=-C \ln x+\int \frac{1}{Q(x)} d x=-C \ln x+\sum_{i=1}^{n} \frac{\ln \left(x-\alpha_{i}\right)}{Q^{\prime}\left(\alpha_{i}\right)}$, que no es una función racional para ningún valor de $C$ (recuerde que dijimos que cada $\alpha_{i} \neq 0$ ).

A la luz de este resultado, $\int \frac{\ln x}{x} d x$ es elemental pero $\int \frac{\ln x}{x+1} d x$ no es elemental, ni tampoco $\int \frac{\ln x}{x^{2}+1} d x$, etc.

Ejemplo 1.20

$\int \operatorname{arcsec}^{2}(x) d x$ no es elemental pues, aplicando integración por partes,

$$
\int \operatorname{arcsec}^{2}(x) d x=x \operatorname{arcsen}^{2}(x)+4 \int \frac{\ln t}{t^{2}+1} d t
$$

\subsection{Algoritmo para Integrar una Función Racional}

Como ya sabemos por el algoritmo de Bernoulli, toda función racional $P / Q$ tiene primitiva elemental. En el algoritmo que sigue, el cálculo de esta primitiva no se hace buscando una factorización completa para $Q$, que de por sí puede ser imposible, sino que se divide la integral en una parte racional y una parte logarítmica. La parte racional se obtiene con el método de Hermite y no necesita factorizaciones. La parte logarítmica de la integral se hace con el algoritmo de R/T y usa una factorización 'libre de cuadrados' y el cálculo de raíces de un polinomio especial.

Este algoritmo aparece de manera muy similar en el caso general.

Conocimientos previos: Factorización libre de cuadrados. Desde el punto de vista computacional, para integrar una función racional, se trata de factorizar el denominador pero de una manera menos demandante que la factorización total. Esta factorización se llama 'libre de cuadrados'. Esta factorización 'empaqueta' los factores de $Q$ que tiene la misma multiplicidad, en un solo factor y solo requiere división de polinomios y cálculo del máximo común divisor.

Un polinomios es 'libre de cuadrados' si no tiene factores repetidos. Si un polinomio tiene factores repetidos, entonces estos factores vuelven a aparecer en la derivada: $\operatorname{Si} P=A^{2} B$ entonces $P^{\prime}=$ 
$2 A A^{\prime} B+A^{2} B^{\prime}=A\left(2 A^{\prime} B+A B^{\prime}\right)$. Por tanto, $P$ es libre de cuadrados si $\operatorname{mcd}\left(P, P^{\prime}\right)=1$.

\section{Definición 1.1}

a) Un polinomios es 'libre de cuadrados' si no tiene factores repetidos

b) Una factorización 'libre de cuadrados' de $P$ es una factorización $P=\prod_{i=1}^{k}\left(P_{i}\right)^{i}$ (potencias crecientes) donde cada $P_{i}$ es libre de cuadrados y $\operatorname{mcd}\left(P_{i}, P_{j}\right)=1$ si $i \neq j$. Algunos $P_{i}$ pueden ser 1.

\section{Teorema 1.5}

Un polinomio $P \in \mathbb{Q}[x]$ es libre de cuadrados si y sólo si $\operatorname{mcd}\left(P, P^{\prime}\right)=1$

Prueba. $(\Longleftarrow)$ Si $P$ tiene algún factor repetido, entonces $P=Q^{n} D$ con $n>1$. Por tanto $P^{\prime}=n Q^{n-1} D+$ $D^{\prime} Q$. Como $Q$ es un factor común entre $P$ y $P^{\prime}$, esto contradice la hipótesis de que $\operatorname{mcd}\left(P, P^{\prime}\right)=1$.

$(\Longrightarrow)$ Si $P$ es 'libre de cuadrados' (sin factores repetidos) y si $P$ tiene la factorización irreducible

$$
P=Q_{1} Q_{2} \cdots Q_{n} \text { con todos los } Q_{i} \text { distintos, }
$$

entonces

$$
P^{\prime}=Q_{1}^{\prime} Q_{2} \cdots Q_{n}+Q_{1} Q_{2}^{\prime} Q_{3} \cdots Q_{n}+\ldots+Q_{1} Q_{2} \cdots Q_{n-1} Q_{n}^{\prime}
$$

Supongamos que $\operatorname{mcd}\left(P, P^{\prime}\right)>1$ y sea $D \in \mathbb{Q}[x]$ no trivial con $D \mid P$ y $D \mid P^{\prime}$, entonces como $D \in \mathbb{Q}[x]$, $D$ debe ser un múltiplo de algún $Q_{i}$. Por tanto podemos asumir que algún $Q_{i}$ divide a ambos $P$ y $P^{\prime}$. Entonces $Q_{i}$ divide todos los sumandos de $P^{\prime}$ donde él aparece y como divide a $P^{\prime}$, deberá también dividir al sumando $Q_{1} \cdots Q_{i}^{\prime} \cdots Q_{n}$, por tanto debe dividir a $Q_{i}^{\prime}$, pero esto es imposible pues $Q_{i}^{\prime}$ tiene menor grado que $Q_{i}$.

Note que hay una diferencia entre ser 'libre de cuadrados' y la 'factorización libre de cuadrados'.

Algoritmo para obtener la 'factorización libre de cuadrados'. Para ver la manera en que se obtienen los factores $P_{i}$ 'libres de cuadrados' en la factorización 'libre de cuadrados' de $P$, hagamos un cálculo pequeño: Supongamos que la factorización libre de cuadrados de $P$ es $P=P_{1} P_{2}^{2} P_{3}^{3}$ (hasta ahora desconocida) entonces queremos calcular estos tres $P_{i}$. Primero calculamos $P^{\prime}$ y factorizamos,

$$
P^{\prime}=P_{2} P_{3}^{2}\left[P_{2} P_{3} P_{1}^{\prime}+2 P_{1} P_{3} P_{2}^{\prime}+3 P_{1} P_{2} P_{3}^{\prime}\right]
$$

Entonces $A_{1}=\operatorname{mcd}\left(P, P^{\prime}\right)=P_{2} P_{3}^{2}$ y $B_{1}=\frac{P}{\operatorname{mcd}\left(P, P^{\prime}\right)}=P_{1} P_{2} P_{3}$. Por tanto, si $C_{1}=\operatorname{mcd}\left(A_{1}, B_{1}\right)=P_{2} P_{3}$, obtenemos

$$
P_{1}=\frac{B_{1}}{C_{1}}
$$


De manera similar obtenemos $P_{2}$ y $P_{3}$.

En general: Primero calculamos $A_{1}=\operatorname{mcd}\left(P, P^{\prime}\right)$ y $B_{1}=\frac{P}{\operatorname{mcd}\left(P, P^{\prime}\right) \text {. }}$

Luego iteramos:

Desde $i=1$ hasta completar el grado de $P$

$$
\begin{aligned}
& C_{i}=\operatorname{mcd}\left(A_{i}, B_{i}\right) \\
& A_{i+1}=\frac{A_{i}}{C_{i}} \\
& B_{i+1}=C_{i} \\
& \therefore P_{i}=\frac{B_{i}}{C_{i}}
\end{aligned}
$$

\section{Ejemplo 1.21}

Obtener la factorización libre de cuadrados de $P(x)=x^{8}-2 x^{6}+2 x^{2}-1$.

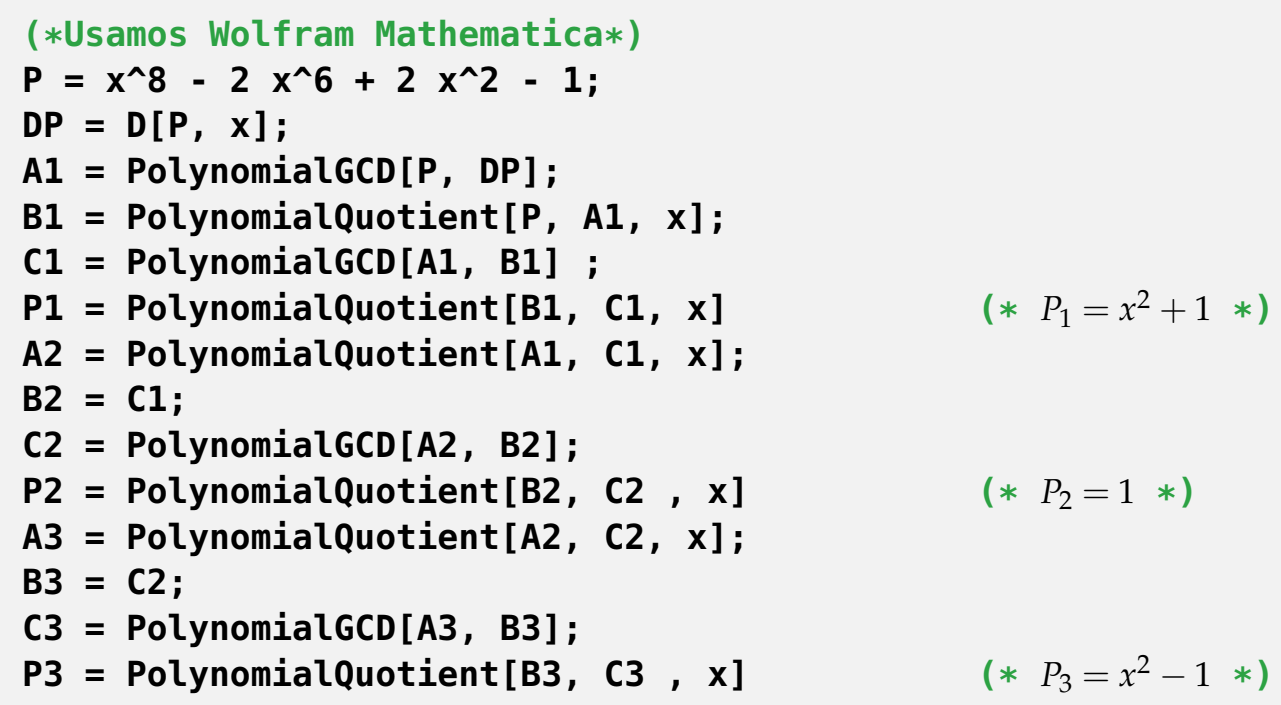

Entonces $P(x)=\left(x^{2}+1\right) \cdot 1^{2} \cdot\left(x^{2}-1\right)^{3}$. 


\subsection{Método de Hermite para obtener la parte racional, 1872.}

Una variante del algoritmo de Bernoulli consiste en usar una factorización completa del denominador en $\mathbb{C}[x]$. Para esta factorización, la descomposición en fracciones parciales no lleva a la integral

$$
\int f=v+\sum_{i=1}^{n} c_{i} \log \left(u_{i}\right)
$$

donde $v, u_{1}, \ldots, u_{n} \in \mathbb{C}(x)$ y cada $c_{i} \in \mathbb{C}$. En esta expresión, $v$ se llama 'la parte racional' de la integral y la suma de logaritmos se llama 'la parte trascendental'. El método de Hermite calcula la parte racional de la integral respecto a la factorización 'libre de cuadrados del denominador' únicamente. La parte 'logarítmica' requiere otro algoritmo. Por ejemplo,

$$
\begin{gathered}
\int \frac{x^{7}-24 x^{4}-4 x^{2}+8 x-8}{x^{8}+6 x^{6}+12 x^{4}+8 x^{2}} d x=\underbrace{\frac{1}{x}+\frac{6 x}{\left(2+x^{2}\right)^{2}}+\frac{3-x}{2+x^{2}}}_{\text {parte racional }}+\int \frac{d x}{x} \\
(145)
\end{gathered}
$$

\section{SUR L'INTÉGRATION DES FRAGTIONS RATIONNELLES;}

PAR M. HERMITE.

\section{Le procédé élémentaire d'intégration des fractions} rationnelles $\frac{F_{1}(x)}{F(x)}$ peut être présenté sous une forme telle, que la résolution de l'équation $F(x)=0$ ne soit plus nécessaire pour le calcul de la partie algébrique de l'intégrale, mais seulement pour en obtenir la partie transcendante. Dans ce but, on mettra d'abord le dénominateur, au moyen de la théorie des racines égales, sous la forme suivante :

$$
\mathbf{F}(\boldsymbol{x})=\mathbf{A}^{a+1} \mathbf{B}^{\beta+1} \ldots \mathbf{L}^{\lambda+1},
$$

Figura 1.2: Método de Hermite, 1872

Pues bien, el método de Hermite hace una reducción del tipo

$$
\int \frac{P}{Q}=\frac{C}{D}+\int \frac{A}{B}
$$


con $P, Q, A, B, C, D \in \mathbb{Q}[x] \operatorname{grado}(A)<\operatorname{grad}(B)$ y $B$ mónico y libre de cuadrados.

$C / D$ es la 'parte racional' de la integral mientras que $\int A / B$ es la 'parte logarítmica' (si esta parte no es nula, requiere extensiones logarítmicas).

Algortimo. El método de Hermite procede como sigue: Primero se normaliza $P / Q$ de tal manera que $\operatorname{mcd}(P, Q)=1$. Esto se logra dividiendo $P$ y $Q$ por el máximo común divisor de ambos. También se requiere que $Q$ sea mónico. Luego aplicamos división euclidiana a $P$ y $Q$ y obtenemos $L, R \in \mathbb{Q}[x]$ tal que $P=Q \cdot L+R$ con $R=0$ o grado $(R)<\operatorname{grado}(Q)$. Obtenemos,

$$
\int \frac{P}{Q}=\int L+\int \frac{R}{Q}
$$

Luego, para $\int \frac{R}{Q}$ calculamos la factorización 'libre de cuadrados' de $Q$,

$$
Q=\prod_{i=1}^{k} Q_{i}^{i}
$$

con cada $Q_{i}$ mónico, libre de cuadrados, $\operatorname{mcd}\left(Q_{i}, Q_{j}\right)=1$ si $i \neq j, \operatorname{yg} \operatorname{grado}\left(Q_{i}\right)>0$.

Ahora calculamos la descomposición en fracciones parciales de $R / Q$ respecto a al factorización libre de cuadrados:

$$
\frac{R}{Q}=\sum_{i=1}^{k} \sum_{j=1}^{i} \frac{R_{i j}}{Q_{i}^{j}}
$$

con $R_{i j} \in \mathbb{Q}[x], \operatorname{grado}\left(R_{i j}\right)<\operatorname{grado}\left(Q_{i}\right) \operatorname{si} \operatorname{grado}\left(Q_{i}\right)>0$ y $R_{i j}=0$ si $Q_{i}=1$.

La integral $\int \frac{R}{Q}=\sum_{i=1}^{k} \sum_{j=1}^{i} \int \frac{R_{i j}}{Q_{i}^{j}}$ se puede reducir usando integración por partes y el algoritmo extendido de Euclides de tal manera que cada sumando tenga un denominador 'libre de cuadrados' en vez de una potencia de un factor libre de cuadrados.

Consideremos un sumando particular $\frac{R_{i j}}{Q_{i}^{j}}$ en el que $j>1$. Como $Q_{i}$ es libre de cuadrados, $\operatorname{mcd}\left(Q_{i}, Q_{i}^{\prime}\right)=$ 1. Usando el algoritmo extendido de Euclides determinamos polinomio $t, s \in \mathbb{Q}[x]$ tal que $t \cdot Q_{i}+s$. $Q_{i}^{\prime}=1$. Y en particular se pueden obtener polinomios $S, T \in \mathbb{Q}[x]$ tal que

$$
S \cdot Q_{i}+T \cdot Q_{i}^{\prime}=R_{i j}
$$

donde $\operatorname{grado}(S)<\operatorname{grado}\left(Q_{i}\right)-1$ y $\operatorname{grado}(T)<\operatorname{grado}\left(Q_{i}\right)$. Dividiendo por $Q_{i}^{j}$

$$
\frac{S \cdot Q_{i}}{Q_{i}^{j}}+\frac{T \cdot Q_{i}^{\prime}}{Q_{i}^{j}}=\frac{R_{i j}}{Q_{i}^{j}} \Longrightarrow \int \frac{R_{i j}}{Q_{i}^{j}}=\int \frac{S}{Q_{i}^{j-1}}+\int \frac{T \cdot Q_{i}^{\prime}}{Q_{i}^{j}} .
$$

Ahora aplicamos integración por partes a $\int \frac{T \cdot Q_{i}^{\prime}}{Q_{i}^{j}} \operatorname{con} u=T$ y $v=\frac{-1}{(j-1) Q_{i}^{j-1}}$. Nos queda

$$
\int \frac{T \cdot Q_{i}^{\prime}}{Q_{i}^{j}}=\frac{-T}{(j-1) Q_{i}^{j-1}}+\int \frac{T^{\prime}}{(j-1) Q_{i}^{j-1}}
$$


Entonces tenemos una disminución en el grado del denominador:

$$
\int \frac{R_{i j}}{Q_{i}^{j}}=\frac{-T /(j-1)}{Q_{i}^{j-1}}+\int \frac{S+T^{\prime} /(j-1)}{Q_{i}^{j-1}}
$$

En resumen,

a) Con este proceso, si $j>1$, cada integral $\int \frac{R_{i j}}{Q_{i}^{j}}$ contribuye con $\frac{-T /(j-1)}{Q_{i}^{j-1}}$ a la parte racional de la integral original y la integral $\int \frac{S+T^{\prime} /(j-1)}{Q_{i}^{j-1}}$ podría ser cero y todo el proceso de reducción termina.

b) En el caso de que $j-1=1$, la integral $\int \frac{S+T^{\prime} /(j-1)}{Q_{i}^{j-1}}$ contribuye a la parte logarítmica de la integral original.

c) Si $j-1>1$ se puede aplicar nuevamente el proceso de reducción a $\int \frac{S+T^{\prime} /(j-1)}{Q_{i}^{j-1}}$ hasta que los denominadores restantes queden 'libres de cuadrados' y esto termina con la parte racional de la integral.

\section{Ejemplo 1.22}

Para fines ilustrativos vamos a aplicar el método a la integral $\int \frac{2 x^{7}+16 x^{5}+4 x^{3}}{8 x^{6}+8 x^{4}+2 x^{2}}$ usando Mathematica.

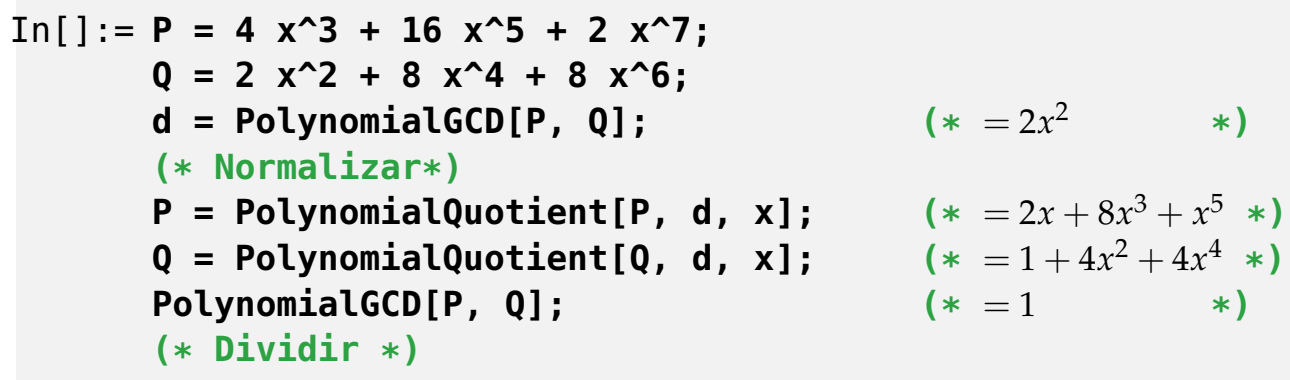


$\{L=\operatorname{PolynomialQuotient}[P, Q, x], R=\operatorname{PolynomialRemainder}[P, Q, x$ ]\} ;

$$
\text { (* } \mathrm{P}=\mathrm{L}+\mathrm{R} / \mathrm{Q} \text { con } \mathrm{L}=\frac{x}{4} \text { y } \mathrm{R}=\frac{7 x}{4}+7
$$

(* Factorización 'libre de cuadrados' de $Q *$ )

FactorSquareFree [Q] ; $\quad\left(* \mathbf{Q}=\left(1+2 x^{2}\right)^{2} *\right)$

(* Expansión en fracciones parciales 'Libre de cuadrados' *)

ApartSquareFree $[\mathbf{R} / \mathbf{Q}] ; \quad\left(* \frac{7 x}{2\left(1+2 x^{2}\right)}-\frac{7 x}{4\left(1+2 x^{2}\right)^{2}} *\right)$

$\mathrm{R} 11=7 \mathrm{x} / 2$;

$\mathrm{R} 12=-7 \mathrm{x} / 4$;

Q1 = $1+2 \mathrm{x}^{\wedge} 2$;

( $*$ Solo tenemos el caso $\mathrm{j}=2 *$ )

(* Calcular S y T con algoritmo extendido de Euclides para Q1 y Q1'*)

Q1p $=D[Q 1, x]$;

$\{d,\{s, t\}\}=$ PolynomialExtendedGCD $[Q 1, Q 1 p, x]$;

$\{\mathbf{S}=\mathbf{S} * \mathbf{R} 12, \mathbf{T}=\mathbf{t} * \mathbf{R} 12\} ; \quad$ (* $\mathrm{T}=\frac{7 x^{2}}{8} \quad *$ )

(* Resultado: Parte racional $*$ )

PRacional=Integrate $[L, x]-T / Q 1$

(* Parte logarítmica *)

Integrate[R11/Q1] + Integrate[(S+Tp)/Q1] ;

out []$=\frac{x^{2}}{8}-\frac{7 x^{2}}{8\left(2 x^{2}+1\right)}$

\section{Ejemplo 1.23}

Aplicar el método de Hermite a la integral $\int \frac{6 x^{5}+6 x^{4}-8 x^{3}-18}{x^{6}-5 x^{4}-8 x^{3}-2 x^{2}+2 x+1}$ usando MATHEMATICA.

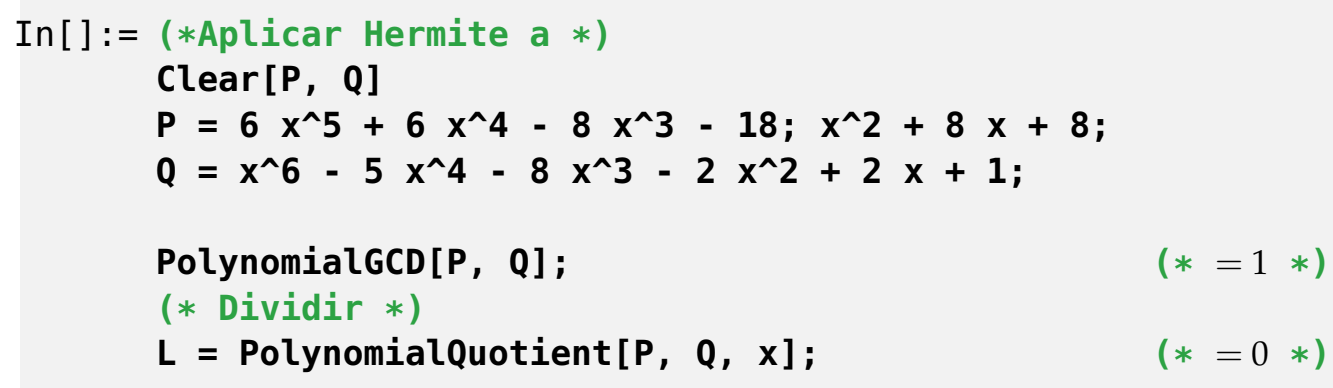




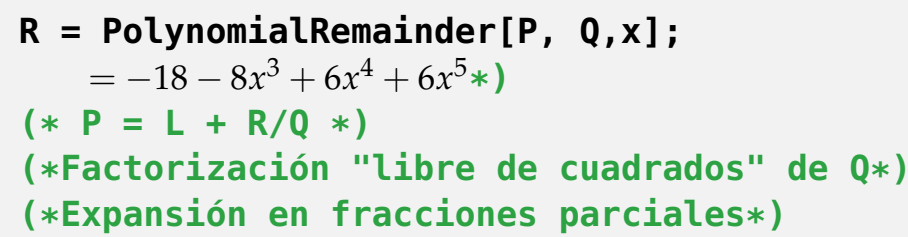

ApartSquareFree $[\mathbf{R} / \mathbf{Q}] ; \quad\left(*=\frac{2\left(-9-4 x^{3}+3 x^{4}+3 x^{5}\right)}{1+2 x-2 x^{2}-8 x^{3}-5 x^{4}+x^{6}} *\right)$

( $* j=1$. La integral solo tiene parte logarítmica $*$ )

\subsection{Algoritmo de Rothstein/Trager para obtener la parte logarítmica.}

Después de aplicar el método de Hermite, podemos suponer que en la integral solo quedan fracciones propias $R_{i j} / Q_{i}$ con $Q_{i}$ mónico y libre de cuadrados. Las integrales de estas fracciones contribuyen a la 'parte logarítmica' de la integral y como tal, deben tener la forma

$$
\int \frac{R}{Q}=\sum_{i=1}^{n} c_{i} \log \left(v_{i}\right)
$$

donde los $v_{i}$ se pueden asumir 'libres de cuadrados' y primos relativos y los $c_{i}$ son todos distintos. Esto se puede suponer porque podemos usar las propiedades del logaritmo para separar o agrupar,

$$
c_{1} \log \left(v_{1} v_{2}\right)+c_{2} \log \left(v_{1} v_{3}\right)=\left(c_{1}+c_{2}\right) \log v_{1}+c_{1} \log v_{2}+c_{2} \log v_{3}
$$

Rothstein $([27,1977])$ y Trager descubrieron de manera independiente que en la ecuación (1.7), cada $v_{i}=\operatorname{mcd}\left(R-c_{i} Q^{\prime}, Q\right)$ y los $c_{i}$ son los números tales que $\operatorname{mcd}\left(R-c_{i} Q^{\prime}, Q\right) \neq 0$, esto es equivalente a que 'el resultante' $\operatorname{res}_{x}\left(R-c_{i} Q^{\prime}, Q\right)=0$. Este 'resultante' es un polinomio en $c$ con grado menor o igual que el de $Q$ y con raíces posiblemente repetidas, por tanto se puede factorizar de manera rápida usando factorización 'libre de cuadrados'.

\section{Teorema 1.6}

(Rothstein/Trager, 1977). Sean $R, Q \in \mathbb{Q}[x] \operatorname{con} \operatorname{mcd}(R, Q)=1$ tal que $Q$ es mónico y libre de cuadrados. Sea $S$ el conjunto de ceros distintos del polinomio res $x\left(R-c Q^{\prime}, Q\right)$. Entonces

$$
\int \frac{R}{Q}=\sum_{c \in S} c \log \left(\operatorname{mcd}\left(R-c Q^{\prime}, Q\right)\right)
$$


y esta es la expresión que usa la mínima cantidad de extensiones algebraicas de Q.

Antes de ver un ejemplo, vamos a ver todos los detalles con los que se llega a este resultado.

Cálculo del Resultante: El método de Rothstein/Trager usa el 'Resultante' para calcular la parte logarítmica de la integral. El resultante apareció en el siglo IXX ligado a la solución de problemas de sistemas de ecuaciones no lineales.

Matriz de Sylvester. Sean $A(x), B(x) \in F[x]$ con $A(x)=\sum_{i=0}^{m} a_{i} x^{i}$ y $B(x)=\sum_{i=0}^{n} b_{i} x^{i}$. La matriz de Sylvester de $A$ y $B$ es la matriz $(m+n) \times(m+n)$

$$
M=\left(\begin{array}{ccccccc}
a_{m} & a_{m-1} & \cdots & a_{1} & a_{0} & & \\
& a_{m} & a_{m-1} & \cdots & a_{1} & a_{0} & \\
& & \cdots & & \cdots & \cdots & \\
& & & a_{m} & \cdots & \cdots & a_{0} \\
b_{n} & b_{n-1} & \cdots & b_{1} & b_{0} & & \\
& b_{n} & b_{n-1} & \cdots & b_{1} & b_{0} & \\
& & \cdots & & \cdots & \cdots & \\
& & & b_{n} & \cdots & \cdots & b_{0}
\end{array}\right)
$$

donde las entradas que no se muestran son cero.

\section{Ejemplo 1.24}

$$
\begin{aligned}
& \text { Si } A(x)=a_{3} x^{3}+a_{2} x^{2}+a_{1} x+a_{0} \\
& y \\
& B(x)=b_{2} x^{2}+b_{1} x+b_{0},
\end{aligned} \quad \text { entonces } \quad M=\left(\begin{array}{ccccc}
a_{3} & a_{2} & a_{1} & a_{0} & 0 \\
0 & a_{3} & a_{2} & a_{1} & a_{0} \\
b_{2} & b_{1} & b_{0} & 0 & 0 \\
0 & b_{2} & b_{1} & b_{0} & 0 \\
0 & 0 & b_{2} & b_{1} & b_{0}
\end{array}\right)
$$

\section{Definición 1.2}

El determinante res $(A, B)=\operatorname{det} M$ se llama el resultante de $A$ y $B$. Si se quiere incluir la variable $x$, se escribe $\operatorname{res}_{x}(A, B)$. 
La primera propiedad importante es que 'el resultante' se puede escribir en términos de la raíces de $A$ y $B$.

Teorema 1.7

teores Sean $A, B \in D[x]$ donde $D$ es un dominio integral. Si $A(x)=a_{m} \prod_{i=1}^{m}\left(x-\alpha_{i}\right)$ y $B(x)=$ $b_{m} \prod_{i=1}^{n}\left(x-\beta_{i}\right)$, entonces

$$
\begin{aligned}
\operatorname{res}(A, B) & =(-1)^{m n} b_{n}^{m} \prod_{i=1}^{n} A\left(\beta_{i}\right) \\
& =a^{n} \prod_{i=1}^{n} B\left(\alpha_{i}\right) \\
& =a^{n} b^{n} \prod_{i=1}^{n}\left(\alpha_{i}-\beta_{i}\right)
\end{aligned}
$$

En particular, si $A$ y $B$ tienen un factor $(x-\delta)$ (o una raíz) en común, entonces

$$
\operatorname{res}(A, B)=0 \text { si y sólo si } \operatorname{grado}(\operatorname{mcd}(A, B))>0 .
$$

Como $\mathbb{Q}$ es un campo, $\mathbb{Q}[x]$ es un dominio integral con división ecuclidiana. El 'algoritmo SubResultante' se usa para calcular 'el resultante' ([17, pág 24]).

Fórmula de Rothstein/Trager. Primero necesitamos un lema acerca de la forma de $R$ y $Q$ en términos de los componentes de la primitiva.

Lema 1.0

lema1 Sea $\int \frac{R}{Q}=\sum_{i=1}^{n} c_{i} \log \left(v_{i}\right) \operatorname{con} \operatorname{mcd}(R, Q)=1, Q$ mónico y libres de cuadrados y además

los $v_{i}$ primos relativos, mónicos y libres de cuadrados. Entonces

$$
Q=v_{1} v_{2} \cdots v_{n} \quad \text { y } \quad R=\sum_{i=1}^{n}\left(c_{i} v_{i}^{\prime} \prod_{j \neq i} v_{j}\right)
$$

Prueba. En vez de hacer una prueba formal sobre todos los índices, vamos a ver la idea de la prueba con el caso $n=2$. La generelización después es inmediata. 
Sea

$$
\int \frac{R}{Q}=c_{1} \log v_{1}+c_{2} \log v_{2}
$$

donde $\operatorname{los} v_{1}, v_{2}$ son 'libres de cuadrados' y primos relativos y $c_{1}, c_{2}$ son números distintos.

Entonces, derivando a ambos lados de la ecuación (1.8) se obtiene

$$
\begin{aligned}
\frac{R}{Q} & =c_{1} \frac{v_{1}^{\prime}}{v_{1}}+c_{2} \frac{v_{2}^{\prime}}{v_{2}} \\
& =\frac{c_{1} v_{1}^{\prime} v_{2}+c_{2} v_{2}^{\prime} v_{1}}{v_{1} v_{2}} .
\end{aligned}
$$

de donde,

$$
R v_{1} v_{2}=Q\left(c_{1} v_{1}^{\prime} v_{2}+c_{2} v_{2}^{\prime} v_{1}\right)
$$

Recordemos que $\operatorname{mcd}\left(v_{1}, v_{2}\right)=1$ y cada $v_{i}$ no tiene factores repetidos,es decir, $\operatorname{mcd}\left(v_{1}, v_{1}^{\prime}\right)=1$.

Ahora, tanto $v_{1}$ como $v_{2}$ dividen a $Q\left(c_{1} v_{1}^{\prime} v_{2}+c_{2} v_{2}^{\prime} v_{1}\right)$, pero como no dividen la suma $c_{1} v_{1}^{\prime} v_{2}+c_{2} v_{2}^{\prime} v_{1}$, deben dividir a $Q$, por hipótesis entonces $v_{1} v_{2} \mid Q$. Ahora, como $Q \mid R v_{1} v_{2}$, por hipótesis entonces $Q \mid v_{1} v_{2}$. Como $Q$ y los $v_{i}$ son mónicos se concluye $Q=v_{1} v_{2}$. Finalmente, cancelando $Q$ en la ecuación (1.9) se obtiene la expresión para $R$. Es decir,

$$
Q=v_{1} v_{2} \quad \text { y } \quad R=c_{1} v_{1}^{\prime} v_{2}+c_{2} v_{2}^{\prime} v_{1}
$$

La prueba en el caso general sigue este mismo razonamiento.

Ahora vamos a demostrar, igual con $n=2$, que $v_{i}=\operatorname{mcd}\left(R-c_{i} Q^{\prime}, Q\right)$.

Primero hay que recordar que $\operatorname{mcd}(A-\alpha B, B)=\operatorname{mcd}(A, B)$. Este hecho se usa repetidamente para calcular $v_{1}$ y $v_{2}$. Usando el lema ?? tenemos 


$$
\begin{aligned}
\operatorname{mcd}\left(R-c_{t} Q^{\prime}, v_{i}\right) & =\operatorname{mcd}\left(c_{1} v_{1}^{\prime} v_{2}+c_{2} v_{2}^{\prime} v_{1}-c_{t}\left(v_{1}^{\prime} v_{2}+v_{1} v_{2}^{\prime}\right), v_{i}\right) \\
& =\operatorname{mcd}\left(\left(c_{1}-c_{t}\right) v_{1}^{\prime} v_{2}+\left(c_{2}-c_{t}\right) v_{2}^{\prime} v_{1}, v_{i}\right) \\
& =\left\{\begin{array}{l}
\operatorname{mcd}\left(\left(c_{1}-c_{t}\right) v_{1}^{\prime} v_{2}+\left(c_{2}-c_{t}\right) v_{2}^{\prime} v_{1}, v_{1}\right) \text { si } i=1 \\
\operatorname{mcd}\left(\left(c_{2}-c_{t}\right) v_{2}^{\prime} v_{1}+\left(c_{1}-c_{t}\right) v_{1}^{\prime} v_{2}, v_{2}\right) \text { si } i=2
\end{array}\right. \\
& =\left\{\begin{array} { l l l } 
{ \operatorname { m c d } ( ( c _ { 1 } - c _ { t } ) v _ { 1 } ^ { \prime } v _ { 2 } , } & { v _ { 1 } ) } & { \text { si } i = 1 } \\
{ \operatorname { m c d } ( ( c _ { 2 } - c _ { t } ) v _ { 2 } ^ { \prime } v _ { 1 } , } & { v _ { 2 } ) } & { \text { si } i = 2 } \\
{ \operatorname { m c d } ( 0 , v _ { 1 } ) = v _ { 1 } } & { \text { si } } & { t = 1 } \\
{ \operatorname { m c d } ( 0 , v _ { 2 } ) = v _ { 2 } } & { \text { si } t = 2 }
\end{array} \quad \left\{\begin{array}{ll}
1 & \text { si } t \neq 1 \\
1 & \text { si } t \neq 2
\end{array}\right.\right.
\end{aligned}
$$

Finalmente, si se conocen los $c_{1}$ y $c_{2}$, entonces

$$
\begin{aligned}
\operatorname{mcd}\left(R-c_{i} Q^{\prime}, Q\right)=\operatorname{mcd}\left(R-c_{i} Q^{\prime}, v_{1} v_{2}\right) & =\operatorname{mcd}\left(R-c_{i} Q^{\prime}, v_{1}\right) \operatorname{mcd}\left(R-c_{i} Q^{\prime}, v_{2}\right) \\
& = \begin{cases}v_{1} \cdot 1=v_{1} & \text { si } i=1 \\
1 \cdot v_{2}=v_{2} & \text { si } i=2\end{cases}
\end{aligned}
$$

Para el cálculo de los $c_{i}$ solo hay que observar que estos números $c$ son precisamente los números tales que

$$
\operatorname{mcd}\left(R-c Q^{\prime}, Q\right) \neq 1
$$

pero, por el teorema ??, estos las raíces del polinomio res $_{x}\left(R-c Q^{\prime}, Q\right)=0$.

De nuevo, la demostración del caso general sigue el mismo razonamiento.

\section{Ejemplo 1.25}

En el ejemplo 1.22 calculamos la parte racional de la integral

$$
\int \frac{2 x^{7}+16 x^{5}+4 x^{3}}{8 x^{6}+8 x^{4}+2 x^{2}}
$$

con el método de Hermite. Ahora vamos a completar el cálculo con la parte logarítmica. Teníamos en el ejemplo 1.22 


$$
\int \frac{2 x^{7}+16 x^{5}+4 x^{3}}{8 x^{6}+8 x^{4}+2 x^{2}}=\frac{x^{2}}{8}-\frac{7 x^{2}}{8\left(2 x^{2}+1\right)}+\underbrace{\int \frac{7 x / 2}{1+2 x^{2}}+\int \frac{0}{1+2 x^{2}}}_{\text {Parte logartmica }}
$$

Solo debemos calcular $\int \frac{7 x / 2}{1+2 x^{2}}$ con el algoritmo de Rothsteinh/Trager.

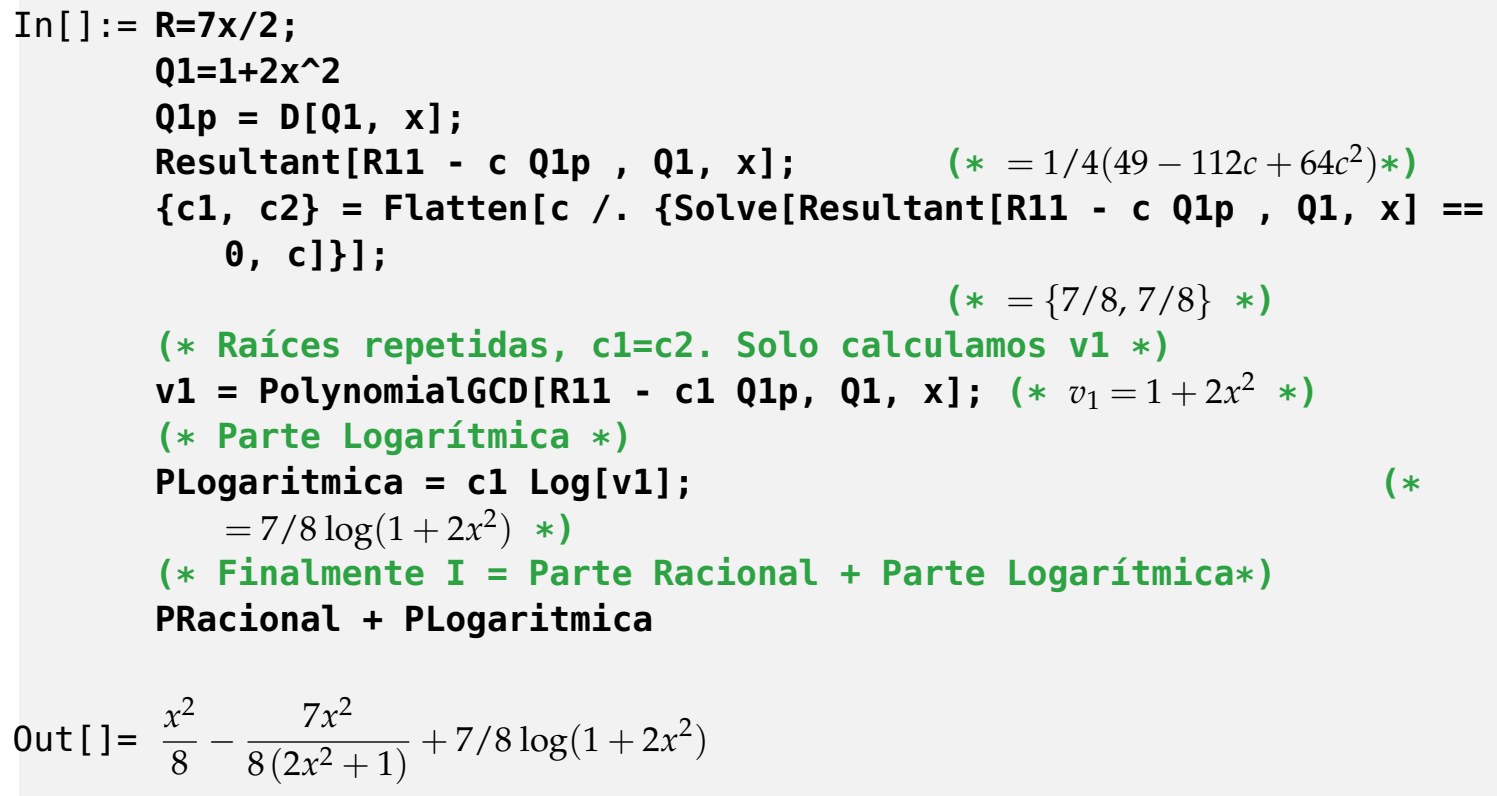

\section{Ejemplo 1.26}

Ya habíamos visto, en el ejemplo 1.23, que la integral

$$
\int \frac{7 x^{1} 3+10 x^{8}+4 x^{7}-7 x^{6}-4 x^{3}-4 x^{2}+3 x+3}{x^{1} 4-2 x^{8}-2 x^{7}-2 x^{4}-4 x^{3}-x^{2}+2 x+1} d x
$$

solo tiene la parte logarítmica. Bien, la vamos a calcular ahora.

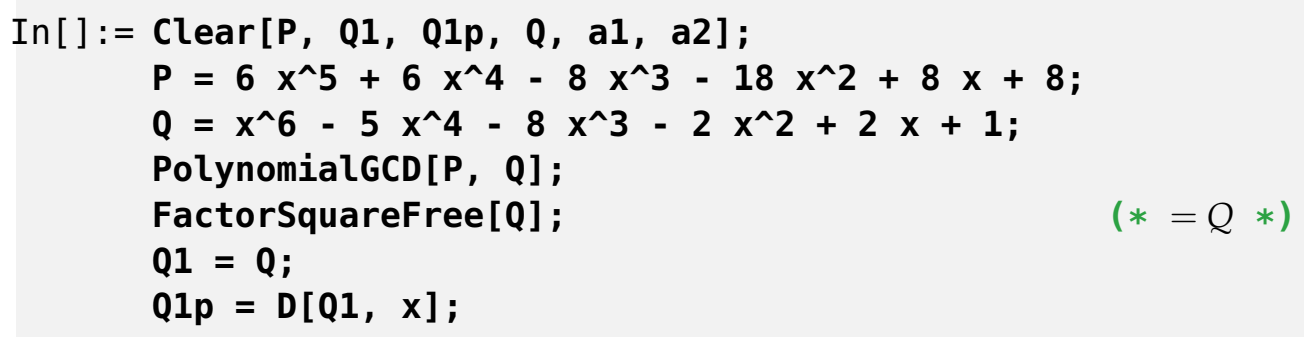




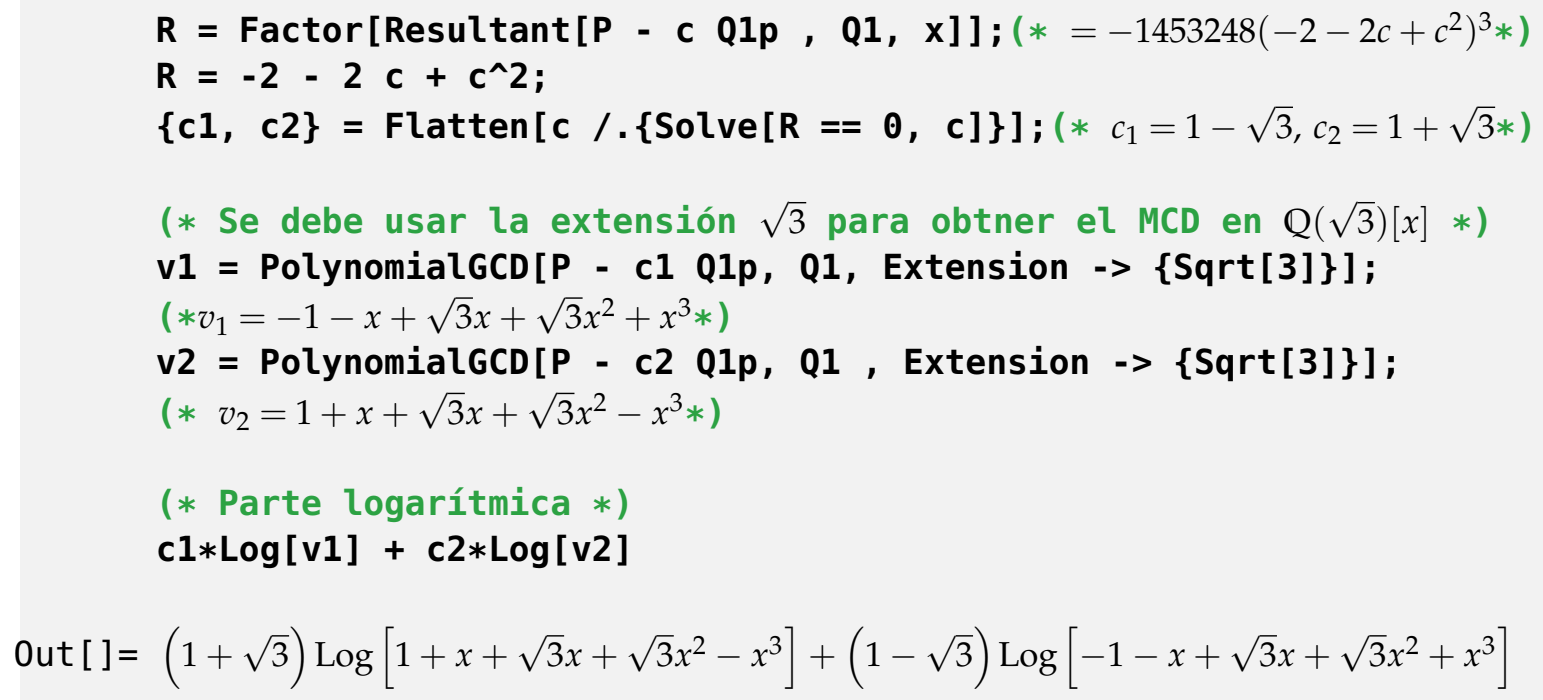

\subsection{4 Álgebra diferencial y el algoritmo de Risch}

Un hecho notable en integración simbólica es que toda la teoría se puede poner en términos algebraicos, sin usar procesos de límite. A mediados del siglo XX, J. Ritt inventó el Álgebra Diferencial que es un marco apropiado para tratar el problema de integrar una función en términos finitos. En 1968, M. Rosenlicht publicó la primera versión puramente algebraica del teorema de Liouvile usando Álgebra Diferencial y R. Risch, en 1970, especificó un algoritmo para el teorema. El algoritmo reduce el problema de integración a un problema de decisión, pero no ha sido implementado totalmente en ningún sistema por lo complejo que se puede volver en algunos casos.

\section{Definición 1.3}

Un campo diferencial es un campo $\mathbb{F}$ de característica 0 en el cual se ha definido una aplicación $D: \mathbb{F} \rightarrow \mathbb{F}$ tal que para todo $f, g \in F$ se cumple

$$
\begin{gathered}
D(f+g)=D(f)+D(g) \\
D(f \cdot g)=f \cdot D(g)+g \cdot D(f)
\end{gathered}
$$

La aplicación $D$ se llama 'operador diferencial' 


\section{Teorema 1.8}

Sea $D$ un operador diferencial en un campo diferencial $\mathbb{F}$, entonces se cumple

a) $D(0)=D(1)=0$

b) $D(-f)=-D(f)$ para toda $f \in \mathbb{F}$

c) $D\left(\frac{f}{g}\right)=\frac{g \cdot D(f)-f \cdot D(g)}{g^{2}}$ para toda $f, g \in \mathbb{F}$ con $g \neq 0$

d) $D\left(f^{n}\right)=n f^{n-1} D(f)$ para toda $n \in \mathbb{Z}, f \in \mathbb{F}$ con $f \neq 0$

Prueba. La demostración usa la definición 1.3 y es enteramente algebraica.

a) $D(0)=D(0+0)=D(0)+D(0)$ por (1.10). Por tanto $D(0)=0$.

$D(1)=D(1 \cdot 1)=1 \cdot D(1)+1 \cdot D(1)$ por (1.11). Por tanto $D(1)=0$.

b) $D(0)=D(f+(-f))=D(f)+D(-f)=0$, entonces $D(-f)=-D(f)$.

c) Como $0=D(1)=D(g \cdot 1 / g)=g \cdot D(1 / g)+1 / g \cdot D(g)$, entonces $D\left(\frac{1}{g}\right)=-\frac{D(g)}{g^{2}}$ si $g \neq 0$.

Ahora, $D\left(\frac{f}{g}\right)=f \cdot D(1 / g)+1 / g \cdot D(f)=\frac{-f \cdot D(g)}{g^{2}}+\frac{D(f)}{g}=\frac{g \cdot D(f)-f \cdot D(g)}{g^{2}}$

d) Se prueba por inducción sobre $n$.

Interludio: Extensiones de campo

Para lo que sigue necesitamos recordar un poco de 'extensiones de campo'. Sea $\mathbb{F}$ un campo. Si $\mathbb{F}$ es un subcampo de $\mathbb{E}$ entonces $\mathbb{E}$ es 'una extensión de campo' de $\mathbb{F}$.

$\mathbb{E}$ se puede ver como un espacio vectorial sobre $\mathbb{F}$ y como tal, puede tener dimensión finita o infinta. Si la dimensión es finita, $\mathbb{E}$ se dice 'extensión finita' de $\mathbb{F}$.

Por ejemplo, el campo $\mathbb{Q}(\sqrt{2})=\{a+b \sqrt{2},: a, b \in \mathbb{Q}\}$ es una extensión de $\mathbb{Q}$.

Sea $\alpha \in \mathbb{E}, \alpha$ es algebraico sobre $\mathbb{F}$ si $\alpha$ es raíz de un polinomio $P \neq 0, P \in \mathbb{F}[x]$. Si $\alpha$ no es algebraico, es trascendental. 
Sea $\alpha$ tascendente sobre $\mathbb{F}$. Entonces $\mathbb{F}(\alpha)=\left\{\frac{f(\alpha)}{g(\alpha)}: f, g \in \mathbb{F}[x], g(\alpha) \neq 0\right\} . \mathbb{F}(\alpha)$ es el más pequeño subcampo de $\mathbb{E}$ que contiene a $\mathbb{F}$ y a $\alpha$. A $\mathbb{F}(\alpha)$ se le llama una 'extensión simple' de $\mathbb{F}$.

Por ejemplo, sea $\theta=\log x$. Entonces $\mathbb{Q}(x, \theta)$ es una extensión del conjunto de funciones racionales $\mathbb{Q}(x), \mathrm{y}$
a) $\frac{x+x^{2} \log ^{2} x}{x}=\frac{x+x^{2} \theta^{2}}{x} \in \mathbb{Q}(x, \theta)$
b) $\frac{x+\sqrt{2} \log ^{2} x}{x+\log x}=\frac{x+\sqrt{2} \theta^{2}}{x+\theta} \in \mathbb{Q}(\sqrt{2})(x, \theta)$

Si $\alpha_{1}, \alpha_{2}, \ldots, \alpha_{k}$ son elementos de $\mathbb{E}$, entonces

$$
\mathbb{F} \subseteq \mathbb{F}\left(\alpha_{1}\right) \subseteq \mathbb{F}\left(\alpha_{1}, \alpha_{2}\right)=\mathbb{F}\left(\alpha_{1}\right)\left(\alpha_{2}\right) \subseteq \ldots \subseteq \mathbb{F}\left(\alpha_{1}, \ldots, \alpha_{k}\right)=\mathbb{F}\left(\alpha_{1}, \ldots, \alpha_{k-1}\right)\left(\alpha_{k}\right)
$$

Sea $\mathbb{F}$ un subcampo de $\mathbb{E}$ y sea $\alpha \in \mathbb{E}$ algebraico sobre $\mathbb{F}$. El (único) polinomio mónico e irreducible $P \in \mathbb{F}[x]$ tal que $P(\alpha)=0$, se llama 'polinomio mínimo' de $\alpha$ sobre $\mathbb{F}$. Si $\operatorname{grado}(P(x))=n \geq 1$ entonces

$$
F(\alpha)=\mathbb{F}[\alpha]=\left\{c_{0}+c_{1} \alpha+\cdots+c_{n-1} \alpha^{n-1}: c_{i} \in \mathbb{F} .\right\}
$$

Extensión diferencial de un campo diferencial. Si $\mathbb{F}, \mathbb{E}$ son campos diferenciales con operadores diferenciales $D_{F}$ y $D_{G}$ respectivamente, entonces decimos que $\mathbb{E}$ es una 'extensión diferencial' de $\mathbb{F}$ si $\mathbb{E}$ es una extensión (de campo) de $\mathbb{F}$ y si

$$
D_{F}(f)=D_{G}(f) \text { para todo } f \in \mathbb{F} .
$$

Homomorfismos diferenciales. Si $\mathbb{F}, \mathbb{E}$ son campos diferenciales con operadores diferenciales $D_{F}$ y $D_{G}$ respectivamente, la aplicación $\phi: \mathbb{F} \rightarrow \mathbb{E}$ es un 'homomorfismos diferencial' si $\phi$ es un homomorfismo de campo y

$$
\phi\left(D_{F}(f)\right)=D_{G}(\phi(f)) \text { para todo } f \in \mathbb{F}
$$

Si $\mathbb{E}$ es una extensión de $\mathbb{F}$, existe un homomorfismo inyectivo $\phi$, de $\mathbb{E}$ en $\mathbb{F}$ tal que $\phi\left(D_{F}(f)\right)=$ $D_{G}(\phi(f))$ para todo $f \in \mathbb{F}$. Esto dice que existe un subconjunto en $\mathbb{E}$, isomorfo a $\mathbb{F}$ y las derivadas coinciden en este subconjunto.

Campo de constantes. Sea $\mathbb{F}$ un campo diferencial con operador diferencial $D$. El campo de constantes de $\mathbb{F}$ es el subcampo $\mathbb{K}$ definido como

$$
\mathbb{K}=\{c \in \mathbb{F}: D(c)=0\}
$$


Extensiones. Una función racional $f \in \mathbb{Q}(x)$ 'es integrable en $\mathbb{Q}(x)^{\prime}$ si existe $g \in \mathbb{Q}(x)$ tal que $D(g)=f$. Ya sabemos que $1 / x \in \mathbb{Q}(x)$ pero no existe $g \in \mathbb{Q}(x)$ tal que $D(g)=1 / x$. Por lo tanto, para expresar la antiderivada de una función racional podría ser necesario extender el campo $\mathbb{Q}(x)$ con nuevas funciones. Como ya sospechamos, solo se necesitan logaritmos.

\section{Ejemplo 1.27}

Consideremos el campo de funciones racionales $\mathbb{Q}(x)$. Si $D(f)=f^{\prime}$ (es decir, la derivada ordinaria) entonces $\mathbb{Q}(x)$ es un campo diferencial. En este caso, el campo de constantes es $\mathbb{K}=\mathbf{Q}$.

$\mathbb{E}=\mathbb{Q}(x, \log x)$ es una extensión de $\mathbb{F}=\mathbb{Q}(x)$. La derivada $D_{E}$ coincide con la derivada $D_{F}$ en $\mathrm{Q}(x)$.

En un contexto general, los logaritmos se definen algebraicamente usando el operador $D$ con una manera que captura la esencia de los logaritmos:

\section{Definición 1.4}

Sea $\mathbb{F}$ un campo diferencial y sea $\mathbb{E}$ una extensión diferencial de $\mathbb{F}$. Un logaritmo sobre $\mathbb{F}$ es un elemento $\theta \in \mathbb{E}$ con la propiedad de que existe $u \in \mathbb{F}$ tal que

$$
D(\theta)=\frac{D(u)}{u} \text { y se escribe } \theta=\log (u)
$$

El problema de integración en términos finitos. Dado un campo diferencial $\mathbb{F}$, el proceso de integración de $f \in \mathbb{F}$ es determinar si existe o no, una extensión de campo $\mathbb{E}$ en la cual existe $g \in \mathbb{E}$ tal que $D(g)=f$.

Consideremos el campo diferencial $\mathbb{F}=\mathbb{Q}(x)$. La función $\frac{1}{x^{2}-2}$ es integrable pues existe una torre de extensiones

$$
\mathbb{F}=\mathbb{Q}(x) \subseteq \mathbb{Q}(\sqrt{2})(x) \subseteq \mathbb{Q}(\sqrt{2})(x, \log (x-\sqrt{2}) \subseteq \mathbb{Q}(\sqrt{2})(x, \log (x-\sqrt{2}, \log (x+\sqrt{2}))=\mathbb{E}
$$


en la cual existe $g \in \mathbb{E}, g=\frac{1}{4} \log (x-\sqrt{2})-\frac{1}{4} \log (x+\sqrt{2})$, tal que $D(g)=f$.

Como se sabe, para cada $f \in \mathbb{Q}(x)$ siempre es posible encontrar una extensión diferencial

$$
\mathbb{E}=\mathbb{Q}\left(\alpha_{1}, \ldots, \alpha_{k}\right)\left(x, \theta_{1}, \theta_{2}, \ldots, \theta_{n}\right)
$$

en la cual exista un elemento $g \in \mathbb{E}$ tal que $D(g)=f$. En este caso, como se sabe, $\alpha_{1}, \ldots, \alpha_{k}$ son números algebraicos y cada $\theta_{i}$ es un logaritmo.

\section{Ejemplo 1.28}
a) $\int \frac{1}{(x+1)^{2}} d x=\frac{-1}{x+1} \in \mathbb{Q}(x)$
b) $\int \frac{1}{x} d x=\log (x) \in \mathbb{Q}(x, \log (x))$
c) $\int \frac{1}{x^{2}-2} d x=\frac{1}{4} \log (x-\sqrt{2})-\frac{1}{4} \log (x+\sqrt{2}) \in \mathbb{Q}(\sqrt{2})(x, \log (x-\sqrt{2}), \log (x+\sqrt{2}))$
d) $\begin{aligned} \int \frac{1-x^{3}}{x^{3}+x} d x= & -x+\log (x)-\frac{1}{2} \log (x-\boldsymbol{i})(x+\boldsymbol{i}) \\ & -\frac{i}{2} \log \frac{x+\boldsymbol{i}}{x-\boldsymbol{i}} \in \mathbb{Q}(i)(x, \log (x), \log (x-\boldsymbol{i}), \log (x+\boldsymbol{i}))\end{aligned}$

En esta sección vamos a trabajar en $\mathbb{K}(x)=\mathbb{Q}(x)$ o en $\mathbb{K}(x)=\mathbb{Q}\left(\alpha_{1}, \ldots, \alpha_{n}\right)\left(y_{1}, y_{2}, \ldots, y_{n}\right)$ donde los $\alpha_{i}$ son números algebraicos y las $y_{i}$ son otras variables. Además, por convención, $D(g)$ se expresará como $g^{\prime}$ y $D^{-1}(f)=\int f$ y además no se incluirá la constante de integración.

Una herramienta que necesitamos es 'integración por partes',

\section{Teorema 1.9}

Si $\mathbb{F}$ es un campo diferencial con derivada $D$, entonces para cualquier $u, v \in \mathbb{F}$,

$$
\int u \cdot D(v)=u \cdot v-\int v \cdot D(u)
$$

La prueba consiste en aplicar $D$ en lado derecho. 


\subsection{Integración de funciones racionales: La parte racional.}

Podemos aplicar el método de Hermite sobre el campo diferencial $\mathbb{K}(x)$ si $\mathbb{K}$ es de característica 0 y si $D(x)=1$.

Recordemos que, siguiendo la presentación del método en la sección 1.12, este método usa operaciones de polinomios para hace la reducción

$$
\int \frac{P}{Q}=\frac{C}{D}+\int \sum_{i=1}^{k} \sum_{j=1}^{i} \frac{R_{i j}}{Q_{i}^{j}}
$$

donde $Q=\prod_{i=1}^{k} Q_{i}^{i}$ es la factorización libre de cuadrados de $Q$. Si $j>1$ entonces existe $S, T \in \mathbb{K}[x]$ tal que

$$
S \cdot Q_{i}+T \cdot Q_{i}^{\prime}=R_{i j}
$$

y, usando integración por partes,

$$
\int \frac{R_{i j}}{Q_{i}}=\frac{-T /(j-1)}{Q_{i}^{j-1}}+\int \frac{S+T^{\prime} /(j-1)}{Q_{i}^{j-1}}
$$

a) $\frac{-T /(j-1)}{Q_{i}^{j-1}}$ es la contribución de cada $\int \frac{R_{i j}}{Q_{i}}$ a la parte racional de la integral.

b) Si $j-1=1, \int \frac{S+T^{\prime} /(j-1)}{Q_{i}^{j-1}}$ contribuye a la parte logarítmica.

c) Si $j-1>1$, se repite el proceso de reducción.

Al final del proceso, obtenemos la parte racional de la integral sumando todas las contribuciones y además obtenemos, sin calcular, la parte logarítmica de la integral.

\subsection{Integración de funciones racionales: La parte logarítmica.}

Después de aplicar el método de Hermite, podemos suponer que en la integral solo quedan fracciones $R_{i j} / Q_{i}$ con $Q_{i}$ mónico y libre de cuadrados.

El método de Rothstein/Trager sigue las mismas líneas presentadas en la sección 1.13. Se enuncia en el teorema que sigue.

¿Se puede saber si una función tiene primitiva elemental? . Walter Mora F.

Derechos Reservados (C) 2015 Revista digital Matemática, Educación e Internet (http://tecdigital.tec.ac.cr/revistamatematica/) 


\section{Teorema 1.10}

Sea $\mathbb{K}^{*}(x)$ un campo diferencial con campo de constantes $\mathbb{K}^{*}$. Sean $P, Q \in \mathbb{K}^{*}[x]$ con $\operatorname{mcd}(P, Q)=1, Q$ mónico, libre de cuadrados y grado $(P)<\operatorname{grado}(Q$. $)$ Supongamos que

$$
\int \frac{P}{Q}=\sum_{i=1}^{n} c_{i} \log \left(v_{i}\right)
$$

donde las $c_{i} \in \mathbb{K}^{*}$ son $n$ constantes distintas no nulas y los $v_{i} \in \mathbb{K}^{*}[x]$ son mónicos, libres de cuadrados y primos relativos dos a dos y de grado positivo. Entonces los $c_{i}$ son las raíces distintas del polinomio

$$
R(c)=\operatorname{res}_{x}\left(P(x)-c Q^{\prime}(x), Q(x)\right) \in \mathbb{K}^{*}[x]
$$

$\mathrm{y}$, una vez calculadas las raíces $c_{i}$, los $v_{i}$ son los polinomios

$$
v_{i}=\operatorname{mcd}\left(P(x)-c_{i} Q^{\prime}(x), Q(x)\right)
$$

Si $\mathbb{K}^{*} \neq \mathbb{Q}$, hay que tener el cuidado de calcular cada $\operatorname{mcd}\left(P(x)-c_{i} Q^{\prime}(x), Q(x)\right)$ en $\mathbb{K}^{*}[x]$ y no en $\mathbb{Q}(x)$.

Un método más eficiente es el algoritmo mejorado de Lazard/Rioboo/Trager, pero para el propósito de este artículo, el método que hemos visto es suficiente.

\subsection{Algoritmo de integración de Risch}

Como ya vimos, integrar una función racional $P(x) / Q(x)$ requiere solo algoritmos para manipulación de polinomios: División de polinomios con resto, cálculo del mcd, factorización de polinomios y solución de ecuaciones.

En el caso general de una función elemental $f$, que podría involucrar funciones tales como log, exp, sen, $\cos , \sqrt[n]{f}$, etc., las herramientas requeridas son algoritmos para manipular polinomios en varias variables. En este caso, las expresiones no racionales que aparecen en $f$ se tratan como símbolos independientes de tal manera que $f$ se manipula como una función racional en varias variables: Los pasos para integrar $f$ son entonces muy parecidos a los pasos que usamos para integrar funciones racionales.

En nuestra nueva notación vamos a definir varias cosas familiares.

¿Se puede saber si una función tiene primitiva elemental? . Walter Mora F.

Derechos Reservados @ 2015 Revista digital Matemática, Educación e Internet (http://tecdigital.tec.ac.cr/revistamatematica/) 


\section{Definición 1.5}

Sea $\mathbb{F}$ un campo diferencial y $\mathbb{E}$ una extensión diferencial de $\mathbb{F}$.

a) $\theta \in \mathbb{E}$ es un 'logaritmo' sobre $\mathbb{F}$ si existe $u \in \mathbb{F}$ tal que $\theta^{\prime}=\frac{u^{\prime}}{u}$. Se escribe $\theta=\log (u)$.

b) $\theta \in \mathbb{E}$ es una 'exponencial' sobre $\mathbb{F}$ si existe $u \in \mathbb{F}$ tal que $\frac{\theta^{\prime}}{\theta}=u^{\prime}$. Se escribe $\theta=\exp (u)$.

c) $\theta \in \mathbb{E}$ es 'algebraico' sobre $\mathbb{F}$ si existe $P \in \mathbb{F}[z]$ tal queda $P(\theta)=0$

d) Si $\theta \in \mathbb{E}$ no es 'algebraico' sobre $\mathbb{F}$, entonces es 'trascendente' sobre $\mathbb{F}$.

e) La extensión diferencial $\mathbb{E}$ se llama 'extensión trascendental elemental' de $\mathbb{F}$ si $\mathbb{E}=$ $\mathbb{F}\left(\theta_{1}, \ldots, \theta_{n}\right)$ donde cada $\theta_{i}$ es trascendental $y$, o es logarítmica o es exponencial sobre el campo $F_{i-1}=\mathbb{F}\left(\theta_{1}, \ldots, \theta_{i-1}\right)$.

f) La extensión diferencial $\mathbb{E}$ se llama 'extensión elemental' de $\mathbb{F}$ si $\mathbb{E}=\mathbb{F}\left(\theta_{1}, \ldots, \theta_{n}\right)$ donde cada $\theta_{i}$ es logarítmica, exponencial o algebraica sobre el campo $\mathbb{F}_{i-1}=F\left(\theta_{1}, \ldots, \theta_{i-1}\right)$.

\section{Definición 1.6}

Sea $\mathbb{K}(x)$ un campo diferencial de funciones racionales sobre un campo de constantes $\mathbb{K} \subseteq \mathbb{C}$. Si $\mathbb{F}$ es una extensión trascendental elemental de $\mathbb{K}(x)$ entonces decimos que $\mathbb{F}$ es un campo de 'funciones elementales trascendentales'. Similarmente, si $\mathbb{F}$ es una extensión elemental de $\mathbb{K}(x)$ entonces $\mathbb{F}$ se llama 'un campo de funciones elementales.'

\section{Ejemplo 1.29}

Sea $\theta_{1}=\log (x)$. $\theta_{1}$ es logarítmica sobre $\mathbb{Q}(x)$.

Sea $\theta_{2}=\exp \left(\theta_{1} / 2\right)$. Como $\frac{\theta_{2}^{\prime}}{\theta_{2}}=\frac{\theta_{2} \cdot \frac{1}{2} \theta_{1}^{\prime}}{\theta_{2}}=\frac{1}{2} \theta_{1}^{\prime} \in \mathbb{Q}\left(x, \theta_{1}\right)$, entonces $\theta_{2}$ es exponencial sobre $\mathbb{Q}\left(x, \theta_{1}\right)$.

$\theta_{2}$ es algebraica sobre $\mathbb{Q}(x)$ pues satisface la ecuación $\theta_{2}^{2}-x=0$

Teoremas de estructura. $\theta$ es un monomio sobre el campo diferencial $\mathbb{F}$ si $\mathbb{F}(\theta)$ y $\mathbb{F}$ tienen el mismo campo de constantes y además $\theta$ es trascendental sobre $\mathbb{F}$ y $\theta$ es, o exponencial o logarítmica sobre $\mathbb{F}$. 
Si $\mathbb{F}(x, \theta)$ es una extensión trascendental de $\mathbb{F}(x)$, las operaciones racionales sobre $\mathbb{F}(x, \theta)$ son equivalentes a ejecutar operaciones aritméticas en el campo de funciones racionales $\mathbb{F}(\theta)$. El problema es saber si una extensión es trascendental (esto puede ser difícil, como en el caso de campos de constantes).

Los teoremas de estructura (ver [5], [17]) establecen requerimientos explícitos para que una nueva extensión sea 'independiente' de las extensiones previas. Estos teoremas establecen los cálculos que se deben hacer para decidir si existe alguna relación algebraica entre los objetos presentes en el campo y el nuevo objeto que se quiere adjuntar. Si no hay relación, el nuevo miembro es un monomio.

Por ejemplo, si $\theta_{1}=\exp (x)$ y $\theta_{2}=\exp (2 x)$, entonces $\theta_{2}=\theta_{2}$, por lo que $\mathbb{Q}\left(x, \theta_{1}, \theta_{2}\right)=\mathbb{Q}\left(x, \theta_{1}\right)$.

En lo que sigue, vamos a suponer que se han aplicado los teoremas de estructura y que la representación de $f$ como $f \in \mathbb{K}\left(\alpha_{1}, \ldots, \alpha_{n}\right)\left(\theta_{1}, \ldots, \theta_{m}\right)$ solo involucra extensiones trascendentes.

Derivadas de funciones elementales. Ahora ya podemos ser más precisos en varias cosas. Un campo de funciones elementales es cualquier extensión del campo de funciones racionales $\mathbb{K}(x)$, finitamente generada, de tal manera que cada extensión sea o logarítmica, exponencial o algebraica.

Desde el punto de vista computacional, es conveniente, como decíamos antes, que $\mathbb{K}$ se de la forma $\mathbb{Q}\left(\alpha_{1}, \alpha_{2}, \ldots, \alpha_{n}\right)$ con cada $\alpha_{i}$ algebraico sobre $\mathbb{Q}$. El número de extensiones $\alpha_{i}$ es el requerido para el problema actual. Por ejemplo, en el caso de $1 /\left(x^{2}-2\right)$ solo se requiere $\alpha_{1}=\sqrt{2}$.

El problema de integración en términos finitos es: $\operatorname{Si} f \in \mathbb{Q}\left(\alpha_{1}, \ldots, \alpha_{n}\right)\left(\theta_{1}, \ldots, \theta_{m}\right)$, determinar extensiones adicionales tal que $g=\int f \in \mathbb{K}\left(\alpha_{1}, \ldots, \alpha_{n+h}\right)\left(\theta_{1}, \ldots, \theta_{m+k}\right)$ y determinar explícitamente $g$,o probar que no existe tal función elemental.

El teorema de Liouville prueba la observación de Laplace (sección 1.5) acerca de que la primitiva de una función no puede incluir ninguna otra exponencial ni ningún otro radical de los que ya figuran en la función. Para probar esto necesitamos establecer primero la acción del operador diferencial $D$ sobre polinomios en $\theta$, donde $\theta$ es trascendental o logarítmica, trascendental o exponencial $\mathrm{u}$ algebraica sobre un campo difeencial $\mathbb{F}$.

\section{Teorema 1.11 (Derivadas)}

Sea $\mathbb{F}$ un campo diferencial y sea $\mathbb{F}(\theta)$ una extensión diferencial de $\mathbb{F}$ con el mismo subcampo de constantes.

a) Si $\theta$ es trascendental o logarítmica sobre $\mathbb{F}$, entonces para cada $P(\theta) \in \mathbb{F}[\theta]$ de grado positivo, $P^{\prime}(\theta) \in \mathbb{F}[\theta]$ y el grado de esta derivada disminuye en 1 si su coeficiente principal es una constante, sino, se mantiene igual. 
b) Si $\theta$ es trascendental o exponencial sobre $\mathbb{F}$, entonces para cada $P(\theta) \in \mathbb{F}[\theta]$ de grado positivo, $P^{\prime}(\theta) \in \mathbb{F}[\theta]$ y el grado de esta derivada se mantiene igual. Además: $P(\theta)$ divide $P^{\prime}(\theta)$ si y sólo si $P(\theta)=H \cdot \theta^{n}$ para alguna $H \in \mathbb{F}$ y $n$ entero.

c) Si $\theta$ es algebraico sobre $\mathbb{F}$ con polinomio mínimo $P(z)=\sum_{i=0}^{N+1} p_{i} z^{i} \in \mathbb{F}[z]$ mónico, entonces

$$
\theta^{\prime}=\frac{d(\theta)}{e(\theta)} \in \mathbb{F}(\theta)
$$

con $d, e \in \mathbb{F}[z] \mathrm{y}$

$$
d(z)=\sum_{i=0}^{N} p_{i}^{\prime} z^{i}, \quad e(z)=\sum_{i=0}^{N}(i+1) p_{i+1} z^{i}
$$

Ahora podemos ver el teorema de Liouville. En este teorema se basan los algoritmos de integración en términos finitos.

Teorema 1.12 (Teorema de Liouville).

Sea $\mathbb{F}$ un campo diferencial con campo de constantes $\mathbb{K}$. Supongamos que para $f \in \mathbb{F}$, existe una extensión elemental $\mathbb{G}$ de $\mathbb{F}$, con el mismo campo de constantes $\mathbb{K}$, y $g \in \mathbb{G}$ tal que $\int f=g$. Entonces existen $v_{0}, v_{1}, \ldots, v_{n} \in \mathbb{F}$ y constantes $c_{0}, c_{1}, \ldots, c_{n} \in \mathbb{K}$ tal que

$$
f=v_{0}^{\prime}+\sum_{i=1}^{m} c_{i} \frac{v_{i}^{\prime}}{v_{i}}, \quad \text { es decir, } \quad \int f=v_{0}+\sum_{i=1}^{m} c_{i} \log \left(v_{i}\right)
$$

El teorema es fácil de interpretar, a al luz del teorema 1.11: Si la primitiva de $f \in \mathbb{F}$ requiere una extensión logarítmica trascendental $\theta$, entonces $f=(c \theta+d)^{\prime} \in \mathbb{F}[\theta]$. La primitiva de $f \in \mathbb{F}$ no requiere una exponencial diferente de las que ya hay en $f$ ni requiere nuevas extensiones algebraicas.

Las primitivas pueden variar en apariencia, pero una de las formas debe encajar con el teorema de Liouville. Por ejemplo, la siguiente primitiva en principio parece que requiere extensiones nuevas diferentes a las que aparecen en la integral,

$$
\int \frac{2 x^{3}-2 x^{2}-1}{(x-1)^{2}} \exp \left(x^{2}\right)=\frac{\exp \left(x^{2}+\log (x) / 2\right)}{2(\sqrt{x}-1)}+\frac{\exp \left(x^{2}+\log (x) / 2\right)}{2(\sqrt{x}+1)}
$$


Las extensiones $\exp \left(x^{2}+\log (x) / 2\right)$ y $\sqrt{x}$ no están en $f$. Pero, $\operatorname{simplificando} \exp \left(x^{2}+\log (x) / 2\right)=$ $\exp \left(x^{2}\right) \sqrt{x}$ y sumando, se obtiene la forma esperada,

$$
\int \frac{2 x^{3}-2 x^{2}-1}{(x-1)^{2}} \exp \left(x^{2}\right)=\frac{x}{x-1} \exp \left(x^{2}\right)
$$

Prueba del teorema de Liouville. La prueba es por inducción y se divide en tres casos, dependiendo de si se requieren extensiones trascendentales logarítmicas, exponenciales o extensiones algebraicas (ver [5], [17], [24]).

La hipótesis de la prueba es que existe $g^{\prime}=f$ con $g \in \mathbb{G}$ y que existen $\theta_{1}, \theta_{2}, \ldots, \theta_{N}$, donde cada $\theta_{i}$ es logarítmica, exponencial o algebraica sobre $\mathbb{F}_{i-1}$, tal que $\mathbb{G}=F\left(\theta_{1}, \theta_{2}, \ldots, \theta_{N}\right)$. Además cada $\mathbb{F}_{i-1}$ tiene el mismo campo de constante $\mathbb{K}$.

La prueba es sobre el número de extensiones $N$ y consiste en demostrar que $f$ tiene la forma establecida por el teorema, ya sea para las extensiones logarítmicas o para establecer que no se necesitan extensiones exponenciales adicionales ni algebraicas de las ya presentes en la integral.

El teorema se cumple para $N=0$ : En efecto, en este caso no hay necesidad de extensiones y $g \in \mathbb{F}$ con lo cual $g^{\prime}=f$ y $m=0$ y $v_{0}=g$.

La hipótesis de inducción es que el teorema se cumple para cualquier número de extensiones $<N$. Para el caso de $N$ extensiones, se puede ver el campo $\mathbb{F}\left(\theta_{1}, \theta_{2}, \ldots, \theta_{N}\right)$ como $\mathbb{F}\left(\theta_{1}\right)\left(\theta_{2}, \ldots, \theta_{N}\right)$. De ahora en adelante ponemos $\theta$ en vez de $\theta_{1}$.

Podemos aplicar el teorema para

$$
f \in \mathbb{F}(\theta) \quad \text { y } \quad g \in \mathbb{F}(\theta)\left(\theta_{2}, \ldots, \theta_{N}\right) .
$$

por hipótesis de inducción, existen $v_{1}(\theta), \ldots, v_{m}(\theta) \in \mathbb{F}(\theta)$ y constantes $c_{1}, c_{2}, \ldots c_{n} \in \mathbb{K}$ tal que

$$
f=v_{0}^{\prime}(\theta)+\sum_{i=1}^{m} c_{i} \frac{v_{i}^{\prime}(\theta)}{v_{i}(\theta)}
$$

Como la prueba completa puede ser extenuante, aquí solo vamos a hacer un caso de la prueba para el caso de extensiones trascendentales y logarítmicas.

Prueba del teorema para el caso de extensiones trascendentales y logarítmicas. Sea $\theta$ es extensión trascendental y logarítmica sobre $\mathbb{F}$. Si fuera necesario, podemos aplicar la regla $\log \left(v_{i} v_{j}\right)=\log v_{i}+$ $\log v_{j}$, y asumir que, para $i=1, \ldots, m$, cada $v_{i}(\theta) \in \mathbb{F}$ o que $v_{i}(\theta)$ es mónico e irreducible en $\mathbb{F}[\theta]$, con grado positivo y además todos estos $v_{i}$ son diferentes y las $c_{i}$ no son nulas. Bien, como $\theta$ es trascendental, $v_{0}(\theta) \in \mathbb{F}(\theta)$. Sea

$$
v_{0}(\theta)=\frac{A(\theta)}{B(\theta)} \text { con } A, B \in \mathbb{F}[\theta], \operatorname{mcd}(A, B)=1 \text { y } B \text { mónico. }
$$


Ahora factorizamos $B(\theta)=\prod_{i=1}^{d} B_{i}(\theta)_{i}^{r}$ con los $B_{i}(\theta)$ distintos, mónicos e irreducibles en $\mathbb{F}(\theta)$ y los $r_{i}$ enteros positivos. Desarrollamos $v_{0}(\theta)$ en fracciones parciales,

$$
v_{0}(\theta)=A_{0}(\theta)+\sum_{i=1}^{d} \sum_{j=1}^{r_{i}} \frac{A_{i j}}{B_{i}(\theta)^{j}} \text { con } A_{0}, A_{i j}, B_{i} \in \mathbb{F}[\theta] \text { y grado } A_{i j}<\text { grado } B_{i}
$$

Entonces, derivando respecto a $x$, la ecuación 1.12 se convierte en

$$
f=A_{0}(\theta)^{\prime}+\sum_{i=1}^{d} \sum_{j=1}^{r_{i}}\left(\frac{A_{i j}(\theta)^{\prime}}{B_{i}(\theta)^{j}}-\frac{j A_{i j}(\theta) B_{i}(\theta)^{\prime}}{B_{i}(\theta)^{j+1}}\right)+\sum_{i=1}^{m} c_{i} \frac{v_{i}(\theta)^{\prime}}{v_{i}(\theta)}
$$

Recordemos que $f$ no depende de $\theta$ (no está presente en $f$ ). Como $\theta$ es logarítmica sobre $\mathbb{F}$, entonces, según el teorema 1.11 , a), para cualquier polinomio $P(\theta)$, mónico e irreducible en $\mathbb{F}[\theta]$ de grado positivo, se tiene $P^{\prime}(\theta) \in \mathbb{F}[\theta]$ y el grado de $P^{\prime}$ es inferior al grado de $P$, por tanto, $P(\theta)$ no divide a $P^{\prime}(\theta)$. Bien, si $P$ es uno de los $B_{i}$ en 1.13 entonces el lado derecho de esta ecuación contiene un término con denominador $P(\theta)^{r_{i}+1}$. Pero este término no puede cancelar con nadie, por lo que debe aparecer en $f$ pero esto contradice el hecho de que $\theta$ no está en $f$. Por tanto la doble suma en 1.13 no debe aparecer.

De la misma manera $P(\theta)$ no puede ser uno de $\operatorname{los} v_{i}(\theta)$ porque no puede cancelar con la derivada $v_{i}(\theta)^{\prime}$ y haría depender $f$ de $\theta$. Así, los $v_{i}$ no dependen de $\theta$.

Entonces $f$ tiene la forma,

$$
f=A_{0}(\theta)^{\prime}+\sum_{i=1}^{m} c_{i} \frac{v_{i}^{\prime}}{v_{i}}
$$

y debe ser que $A_{0}(\theta)^{\prime}$ sea independiente de $\theta$. Por el teorema 1.11, a), solo podría pasar que $A_{0}(\theta)=$ $c \theta+d \in \mathbb{F}[\theta]$ con lo que $f$ tiene la forma

$$
f=d^{\prime}+c \frac{u^{\prime}}{u}+\sum_{i=1}^{m} c_{i} \frac{v_{i}^{\prime}}{v_{i}} \text { con } d, u, v_{i} \in \mathbb{F} \quad \text { y } \quad c, c_{i} \in \mathbb{K},
$$

como se deseaba.

Los otros casos usan el teorema 1.11 tal y como se puede ver en [17], [5] o [24], por ejemplo.

\subsection{Algoritmo de Risch: Caso de extensiones logarítmicas}

Ahora ya podemos pasar a describir el algoritmo de Risch. Como el tema es tan extenso, solo lo vamos a hacer en el caso de extensiones logarítmicas.

El algoritmo de Risch es un proceso de decisión para integrar funciones elementales que están a un campo de funciones trascendentales elementales, es decir,

$$
f \in \mathbb{K}\left(x, \theta_{1}, \theta_{2}, \ldots, \theta_{n}\right)
$$


con $\mathbb{K}$ un subcampo de $\mathbb{C}$ y cada $\theta_{i}$ es trascendente y, $\theta_{i}$ o es logarítmica o es exponencial sobre $\mathbb{K}_{i-1}$. El algoritmo determina si $\int f$ existe como una función elemental, en otro caso, en el camino indica por qué no existe esta primitiva elemental.

Como indicamos antes (teoremas de estructura), suponemos que $\mathbb{K}\left(x, \theta_{1}, \theta_{2}, \ldots, \theta_{n}\right)$ es puramente trascendental. Como cada extensión $\theta$ es un símbolo trascendente, el integrando $f$ puede ser manipulado como una función racional de esos símbolos. Por tanto el algoritmo para funciones trascendentes parece una reminiscencia del algoritmo para integrar funciones racionales. De hecho usamos el método de Hermite para la parte racional y el algoritmo de Rothstein/Trager para la parte logarítmica.

Dada $f \in \mathbb{K}\left(x, \theta_{1}, \theta_{2}, \ldots, \theta_{n}\right), f$ puede ser vista como una función racional en la extensión $\theta=\theta_{n}$, es decir,

$$
f(\theta)=\frac{P(\theta)}{Q(\theta)} \in \mathbb{F}_{n-1}(\theta) \text { con } \mathbb{F}_{n-1}=\mathbb{K}\left(x, \theta_{1}, \theta_{2}, \ldots, \theta_{n-1}\right) .
$$

Además suponemos que $f$ está normalizada de tal manera que $P, Q \in \mathbb{F}_{n-1}(\theta)$ son primos relativos y $Q(\theta)$ mónico. Como antes, la derivación ' es respecto a $x$.

El algoritmo es recursivo. El caso inicial es integración de funciones racionales, en $\mathbb{F}_{0}=\mathbb{K}(x)$, el cual ya está resuelto.

Algoritmo de Risch para extensiones logarítmicas. Sea $\theta$ logarítmica con $\theta^{\prime}=u^{\prime} / u$ con $u \in \mathbb{F}_{n-1}$.

Aplicando división euclidiana a $P(\theta), Q(\theta) \in \mathbb{F}_{n-1}[\theta]$ obtenemos polinomios $C(\theta), R(\theta) \in \mathbb{F}_{n-1}[\theta]$ tal que

$$
P(\theta)=Q(\theta) \cdot C(\theta)+R(\theta) \text { con } R(\theta)=0 \text { o } \operatorname{grado} R(\theta)<\operatorname{grado} Q(\theta) .
$$

Entonces $\int f=\underbrace{\int C(\theta)}_{\text {parte polinomial }}+\underbrace{\int \frac{R(\theta)}{Q(\theta)}}_{\text {parte racional }}$.

Extensiones logarítmicas: Integración de la parte racional. Procedemos con el método de Hermite: Calculamos la factorización libre de cuadrados de $Q(\theta) \in \mathbb{F}_{n-1}[\theta]$,

$$
Q(\theta)=\prod_{i=1}^{k} Q_{i}(\theta)^{i}
$$

con cada $Q_{i}(\theta)$ mónico y libre de cuadrados, $\operatorname{mcd}\left(Q_{i}(\theta), Q_{j}(\theta)\right)=1$ si $i \neq j$ y grado $Q_{k}>0$.

$Q_{i}(\theta)$ es libre de cuadrados en el dominio de polinomios $\mathbb{F}_{n-1}[\theta]$ si $\operatorname{mcd}\left(Q_{i}(\theta), \frac{d}{d \theta} Q_{i}(\theta)\right)=1$. Si $x^{\prime}=1$ para $x \in \mathbb{F}$, entonces esto implica que $\operatorname{mcd}\left(Q_{i}(\theta), Q_{i}(\theta)^{\prime}\right)=1$ ([5, Teorema 12.6]).

Usamos operaciones de polinomios para calcular la expansión en fracciones parciales de $\frac{R(\theta)}{Q(\theta)}$ en la forma 


$$
\frac{R(\theta)}{Q(\theta)}=\sum_{i=1}^{k} \sum_{j=1}^{i} \frac{R_{i j}(\theta)}{Q_{i}^{j}(\theta)}
$$

donde $R_{i j}(\theta) \in \mathbb{F}_{n-1}[\theta]$ y grado $R_{i j}(\theta)<\operatorname{grado} Q_{i}(\theta)$ si grado $Q_{i}>0$ y $R_{i j}(\theta)=0$ si $Q_{i}(\theta)=1$.

Entonces tenemos,

$$
\int \frac{R(\theta)}{Q(\theta)}=\sum_{i=1}^{k} \sum_{j=1}^{i} \int \frac{R_{i j}(\theta)}{Q_{i}^{j}(\theta)}
$$

Ahora procedemos con las integrandos $R_{i j} / Q_{i}^{j}$ no nulos.

Caso $j>1$. Como $\operatorname{mcd}\left(Q_{i}(\theta), Q_{i}(\theta)^{\prime}\right)=1$, existe $s, t \in \mathbb{F}_{n-1}[\theta]$ tal que

$$
S(\theta) \cdot Q_{i}(\theta)+T(\theta) \cdot Q_{i}^{\prime}(\theta)=R_{i j}(\theta)
$$

con grado $S<\operatorname{grado} Q_{i}^{\prime}$ y grado $T<\operatorname{grado} Q_{i}$.

Ahora dividiendo por $Q_{i}(\theta)^{j}$ en la ecuación 1.15 y aplicando integración por partes, se obtiene

$$
\int \frac{R_{i j}(\theta)}{Q_{i}(\theta)}=\frac{-T(\theta) /(j-1)}{Q_{i}^{j-1}(\theta)}+\int \frac{S(\theta)+T(\theta)^{\prime} /(j-1)}{Q_{i}^{j-1}(\theta)}
$$

Si $j-1>1$, se repite el proceso de reducción. Al final del proceso todos los denominadores quedan libres de cuadrados y, podemos escribir la parte racional de la integral como,

$\int \frac{R(\theta)}{Q(\theta)}=\frac{C(\theta)}{D(\theta)}+\int \frac{A(\theta)}{B(\theta)}$ con $A, B, C, D \in \mathbb{F}_{n-1}[\theta]$, grado $A<$ grado $B$ y $B$ mónico y libre de cuadrados

Ahora calculamos $\int \frac{A(\theta)}{B(\theta)}$ con el método de Rothstein/Trager con la variante adecuada para el caso de extensiones logarítmicas.

\section{Teorema 1.13}

Sea $\mathbb{F}$ un campo de funciones elementales con campo de constantes $\mathbb{K}$. Sea $\theta$ trascendental y logarítmica en $\mathbb{F}$ y supongamos que la extensión trascendental elemental $\mathbb{F}(\theta)$ tiene el mismo campo de constantes $\mathbb{K}$. Sea $A(\theta) / B(\theta) \in \mathbb{F}(\theta)$ con $A(\theta), B(\theta) \in \mathbb{F}[\theta]$, $\operatorname{mcd}(A(\theta), B(\theta))=$ 1 , grado $A<\operatorname{grado} B$ y $B$ mónico y libre de cuadrados .

a) $\int \frac{A(\theta)}{B(\theta)}$ es elemental si y sólo si todas las raíces del polinomio $R(z)=\operatorname{res}_{\theta}(A(\theta)-z$. $\left.B(\theta)^{\prime}, B(\theta)\right) \in \mathbb{F}[z]$, son constantes 
b) Si $\int \frac{A(\theta)}{B(\theta)}$ es elemental, entonces $\frac{A(\theta)}{B(\theta)}=\sum_{i=1}^{m} c_{i} \frac{v_{i}(\theta)^{\prime}}{v_{i}(\theta)}$ donde las $c_{i}$ son las raíces distintas de $R(z)$ y donde las $v_{i}(\theta)$ se definen como

$$
v_{i}(\theta)=\operatorname{mcd}\left(A(\theta)-c_{i} \cdot B(\theta)^{\prime}, B(\theta)\right) \in \mathbb{F}\left(c_{1}, c_{2}, \ldots, c_{m}\right)[\theta]
$$

Extensión logarítmica: Integración de la parte polinomial. A diferencia de la parte polinomial en la integral de una función racional simple, el caso polinomial en el caso de extensiones logarítmicas es más laborioso.

Vamos a dar la descripción del algoritmo siguiendo a [5, págs 540-543].

Supongamos que la parte polinomial es

$$
C(\theta)=p_{s} \theta^{s}+p_{s-1} \theta^{s-1}+\cdots+p_{0} \in \mathbb{F}_{n-1}[\theta] \text { y } p_{i} \in \mathbb{F}_{n-1}
$$

Por el principio de Liouville y otras consideraciones, $C(\theta)$ debe tener la forma

$$
p_{s} \theta^{s}+p_{s-1} \theta^{s-1}+\cdots+p_{0}=v_{0}(\theta)^{\prime}+\sum_{i=1}^{m} \frac{v_{i}^{\prime}}{v_{i}}
$$

con $v_{0}(\theta)=q_{s+1} \theta^{s+1}+\cdots+q_{0}$ con $q_{s+1} \in \overline{\mathbb{K}}$ y los otros $q_{i} \in \overline{\mathbb{F}}_{n-1}$. Es decir,

$$
\int p_{s} \theta^{s}+p_{s-1} \theta^{s-1}+\cdots+p_{0}=q_{s+1} \theta^{s+1}+\cdots+q_{0}+\int \sum_{i=1}^{m} \frac{v_{i}^{\prime}}{v_{i}}
$$

Ahora, derivando obtenemos el sistema de ecuaciones

$$
\left\{\begin{aligned}
0 & =q_{s+1}^{\prime} \\
p_{s} & =(s+1) \cdot q_{s+1} \theta^{\prime}+q_{s}^{\prime} \\
& \vdots \\
p_{1} & =2 q_{2} \theta^{\prime}+q_{1}^{\prime} \\
p_{0} & =q_{1} \theta^{\prime}+Q_{0}^{\prime} \text { con } Q_{0}=q_{0}+\sum_{i=1}^{m} c_{i} \log \left(v_{i}\right)
\end{aligned}\right.
$$


En este sistema, los $p_{i} \in \mathbb{F}_{n-1}$ son los conocidos y las incognitas son $q_{s+1} \in \overline{\mathbb{K}}, q_{i} \in \overline{\mathbb{F}}_{n-1}$.

Algoritmo. Aplicando integración en el sistema, obtenemos primero que $q_{s+1}$ es una constante de integración, sea $q_{s+1}=\boldsymbol{b}_{s+1}$ (todavía desconocida!)

Paso $s+1$ ) Integrando en la segunda ecuación, obtenemos

$$
\int p_{s}=(s+1) \boldsymbol{b}_{s+\mathbf{1}} \theta+q_{s}
$$

Ahora el proceso de integración se invoca de manera recursiva para integrar $\int p_{s}$. Para resolver esta ecuación para $\boldsymbol{b}_{\boldsymbol{s + 1}} \in \overline{\mathrm{K}}$ y $q_{s} \in \in \overline{\mathbb{F}}_{n-1}$, se requiere que las siguientes condiciones se cumplan $\int p_{s}:$

$\left.\mathbf{a}^{*}\right) p_{s}$ es elemental

b*) A los sumo hay una extensión logarítmica de $\overline{\mathbb{F}}_{n-1}$ apareciendo en la integral y si hay una debe $\operatorname{ser} \theta=\log (u)$.

Si una de estas dos condiciones falla, entonces la ecuación no tiene solución y se concluye que $\int p_{s}$ no es elemental.

Si las condiciones a) y b) se cumplen, entonces si $\int p_{s}=c_{s} \theta+d_{s}$ con $c_{s} \in \overline{\mathbb{K}}$ y $d_{s} \overline{\mathbb{F}}_{n-1}$, se obtiene

$$
b_{s+1}=\frac{c_{s}}{s+1} \quad \text { y } \quad q_{s}=d_{s}+b_{s}
$$

donde $b_{s} \in \bar{K}$ es una constante de integración no conocida todavía.

Paso $s$ ) Sustituyendo $q_{s}$ en la tercera ecuación, reagrupando e integrando se obtiene,

$$
\int p_{s-1}-s \cdot d_{s} \theta^{\prime}=s \cdot b_{s} \theta+q_{s-1}
$$

Si las condiciones $\mathbf{a}^{*}$ ) y $\mathbf{b}^{*}$ ) se cumplen (sino, $\int p_{s}$ no es elemental y termina el algoritmo), entonces si

$$
\int p_{s-1}-s \cdot d_{s} \theta^{\prime}==c_{s-1} \theta+d_{s-1} \text { con } c_{s-1} \in \overline{\mathbb{K}} \text { y } d_{s-1} \overline{\mathbb{F}}_{n-1}
$$

se obtiene 


$$
\boldsymbol{b}_{\boldsymbol{s}}=\frac{c_{s-1}}{s} \quad \text { y } \quad \boldsymbol{q}_{s-1}=d_{s-1}+b_{s-1}
$$

donde $b_{s-1} \in \overline{\mathbb{K}}$ es una constante de integración no conocida todavía.

\section{$\vdots$}

Paso 2) El proceso descrito se continua hasta llegar hasta la penúltima ecuación donde la solución debe ser de la forma

$$
b_{2}=\frac{c_{1}}{2} \quad y \quad q_{1}=d_{1}+b_{1}
$$

donde $b_{1} \in \overline{\mathrm{K}}$ es una constante de integración no conocida todavía.

Paso 1) Sustituyendo $q_{1}$ en la última ecuación, reagrupando e integrando se obtiene

$$
\int p_{0}-d_{1} \frac{u^{\prime}}{u}=b_{1} \theta+Q_{0}
$$

Si la integral de la izquierda es elemental (sino, $\int p_{s}$ no es elemental y termina el algoritmo) y

$$
\int p_{0}-d_{1} \frac{u^{\prime}}{u}=d_{0}
$$

entonces $b_{1}$ es el coeficiente en $d_{0}$ de $\theta=\log (u)$ y

$$
Q_{0}=d_{0}-b_{1} \log (u)
$$

Con esto hemos calculado (si en el camino no resultó ser $\int p_{s}$ no elemental) la parte polinomial de la integral

$$
\int p_{s}=b_{s+1} \theta^{s+1}+q_{s} \theta^{s}+\ldots+q_{1} \theta+Q_{0}
$$




\section{Ejemplo 1.30}

Apliquemos el algoritmo a el cálculo de la integral $\int \frac{-1+2 x \log ^{3} x+x \log ^{4} x}{x \log ^{2} x} d x$. Solo necesitamos la extensión $\theta=\log x$.

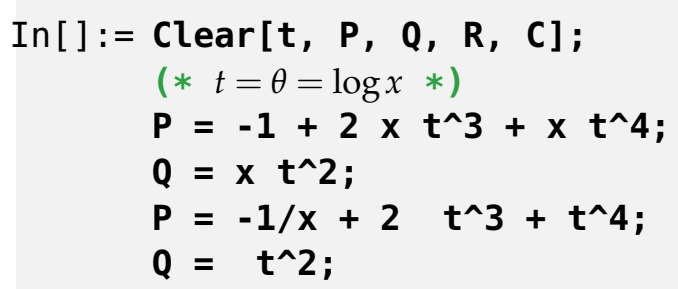

PolynomialGCD [P, Q] ;

$C=$ PolynomialQuotient $[P, Q, t]$;

$\mathbf{R}=\operatorname{PolynomialRemainder}[\mathbf{P}, \mathbf{Q}, \mathbf{t}]$;

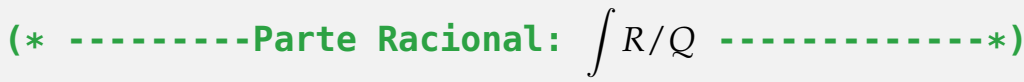

(* - -Hermite - * *)

(*Factorización "libre de cuadrados" de Q*)

FactorSquareFree [Q] ;

y $j=2 *)$

(*Expansión en fracciones parciales*)

ApartSquareFree $[R / Q, t]$;

R11 = -1/x;

Q1 = $t$;

Q1p $=D[Q 1, t]$;

(* Como $j=2 *$ *)

$\{\mathbf{d},\{\mathbf{s}, \mathbf{t}\}\}=$ PolynomialExtendedGCD $[\mathbf{Q} 1, \mathbf{Q 1 p}, \mathbf{t}] ;(* \mathrm{~s}=0, \mathrm{t}=1 *)$

(* $0 \cdot Q 1+t \cdot Q p=1 *)$

$(* 0 \cdot Q 1+t \cdot 1 / x=-1 / x \Longrightarrow T=-1 *)$

$\mathrm{S}=0$;

$\mathrm{T}=-1$;

(* Hermite entrega $*$ )

Hermite $=-\mathrm{T} / \mathrm{Q} \mathbf{1}$;

(* - - Rothstein/Trager: $\left.\int(S+D[T, x]) / Q 1 *\right)$

$(\mathrm{S}+\mathrm{D}[\mathrm{T}, \mathrm{x}]) / \mathrm{Q}$;

RothsteinTrager $=0$;

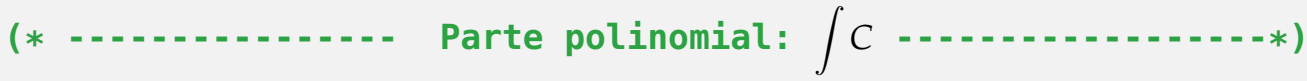

$c=t^{\wedge} 2+2 t ;$

$\mathrm{s}=2 ; \mathrm{p} 2=1 ; \mathrm{p} 1=2 ; \mathrm{p} 0=0$;
$(*=1 *)$

( $\left.*=2 t+t^{2} *\right)$

$(*=-1 / x *)$ 


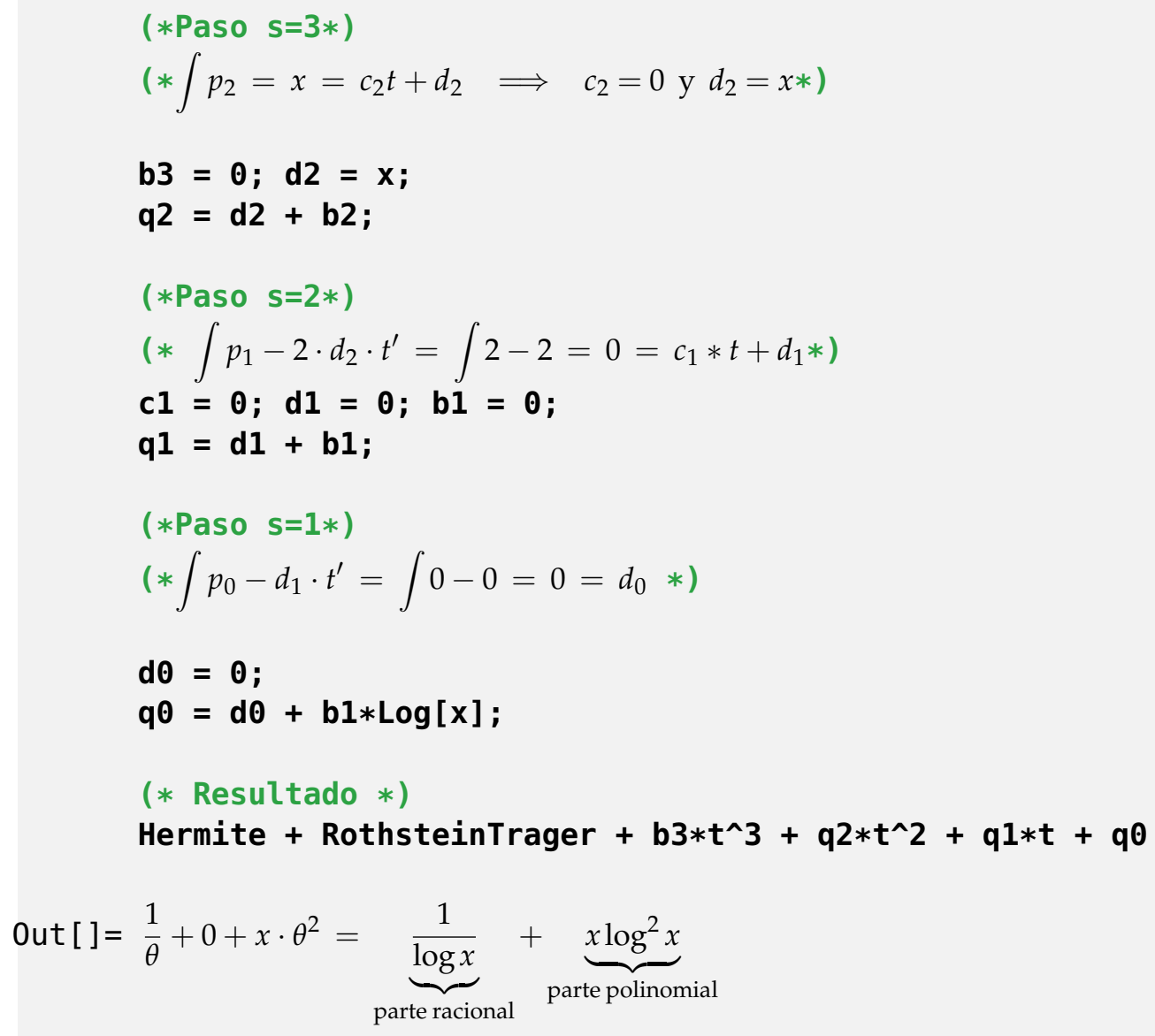

\section{Ejemplo 1.31}

El algoritmo decide si una primitiva no es elemental.

Consideremos la integral $\int \frac{1}{\log x}$. El integrando es $\frac{P(\theta)}{Q(\theta)}=\frac{1}{\theta} \in \mathbb{Q}(x, \theta) \operatorname{con} \theta=\log x$.

Solo debemos aplicar el método de Rothstein/Trager:

$$
R(c)=\operatorname{res}_{\theta}\left(1-\frac{c}{x}, \theta\right)=1-\frac{c}{x} \in \mathbb{Q}(x)[c]
$$

Como $R(c)$ no tiene raíces constantes, la integral no es elemental. 
Como indicabamos, los casos del algoritmo de Risch con las otras extensiones se pueden ver en [16], [17] o [5].

\section{Bibliografía}

[1] S. Abbot. "Understanding Analysis". Springer-Verlag. 2000.

[2] M. Spivak "Calculus" W.A. Benjamin Inc. 1967. http://archive.org/details/Calculus_643. Consultada Julio 6, 2013.

[3] "Oeuvres de P.L. Tchebychef (1899)". http://archive.org/details/117744684_001. Consultada Julio 6, 2013.

[4] Robert H. Risch. "The solution of the problem of integration in finite terms", BulL Amer. Math. Soc., vol. 76, pp. 605-608.

[5] Keith O. Geddes, Stephen R. Czapor, George Labahn. Algorithms for Computer Algebra. Kluwer Academic Publisher. 1992.

[6] G. H. Hardy. "The Integration of Functions of a Single Variable." 2nd ed., Cambridge University Tracts in Mathematics and Mathematical Physics, no. 2, Cambridge, England, 1916. Consultada Julio 6, 2013.http://www. gutenberg.org/ebooks/38993.

[7] P. Laplace. "Oeuvres complétes de Laplace". Tome Septiéme. Gauthier-Villars. 1820. http:// archive.org/stream/theorieanaldepro00laplrich\#page/n193/mode/2up.Consultada Julio 6, 2013.

[8] J. F. Ritt. "Integration in Finite Terms: Liouville's Theory of Elementary Methods". Columbia University Press, New York, 1948.

[9] C. Ivorra. "Funciones sin primitiva elemental". http://www.uv.es/ivorra/Libros/ Primitivas.pdf. Consultada Julio 6, 2013.

[10] N. Piskunov. "Differential and Integral Calculus". Editorial Mir. Moscú. 3ra Ed. 1977. https://ia801507.us.archive.org/33/items/DifferentialAndIntegralCalculus_ 109/N. Piskunov - DifferentialAndIntegralCalculus 1969mir . djvu. Consultada Julio 6, 2013.

[11] J. Moses. "Symbolic Integration". ACM Communications, Vol 14. Number 8. 1971. http: //wwwinst. eecs . berkeley. edu/ cs282/sp02/readings/moses - int. pdf

[12] "Sign function". The Wolfram Functions Site. http://functions.wolfram.com/ ComplexComponents/Sign/introductions/ComplexComplements/ShowAll . html.

Consultada Julio 6, 2013.

[13] J. Pierpont. "Lectures on the theory of functions of a real variable. Vol 1, Ginn and Company, 1905. http://archive.org/details/lecturesonthethe032088mbp. Consultada Julio 6, 2013.

[14] E. A. Marchisotto, G. Zakeri. "An invitation to integration in finite terms". College Mathematics Journal, Vol 25, No. 4. (Sep, 1994).

[15] B. Conrad. "Impossibility theorems for elementary integration." Clay Institute Research Academy Colloquium Series. http://www. claymath.org/programs/outreach/academy/ LectureNotes05/Conrad.pdf

[16] R. Risch. "The Solution of the Problem of Integration in Finite Terms." Bull. Amer. Math. Soc., 1-76, 605-608, 1970.

[17] M. Bronstein. "Symbolic Integration I:Transcendental Functions". 2nd edition. Springer-Verlag, 2005. 
[18] M.W. Ostrogradsky. "De integration des fractions rationelles". Bulletin de la Classe PhysicoMathematiques de l"acadèmie Impériale des Sciences de St. Pétersbourg, IV: 145-167,286-300, 1845. http://books .google.es/books/about/Bulletin_physico_math\%C3\%A9matique. html ?hl=es\&id=DegAAAAAYAAJ\&utm_source=gb - gplus - share. Consultada Agosto 3, 2013.

[19] E. Hermite. "Sur I'ntegration des fractions rationelles". Nouvelles Annales de Mathematiques (2eme serie), 11:145-148, 1872. http://books.google.es/books/about/Nouvelles_annales_ de_math\%C3\%A9matiques. html ?hl=es\&id=GDITAQAAMAAJ\&utm_source=gb - gplus share. Consultada Agosto 3, 2013.

[20] J. Luetzen. "Joseph Liouville 1809-1882". Volume 15 of Studies in the history of mathematics and physical sciences. Springer-Verlag, New York, 1990.

[21] T. Wang. "Techniques on Partial Fractions". Proceedings of the AMATYC 33rd Annual Conference, Minneapolis, Minnesota 2007. American Mathematical Association of Two Year Colleges. http://c.ymcdn.com/sites/www. amatyc.org/resource/resmgr/2007_ Conference_Proceedings/wang07.pdf. Consultada Agosto 3, 2013.

[22] E. Selmer. "The Irreducibility of Certain Trinomials". http://www.mscand.dk/article.php? id=1472. Consultada Agosto 3, 2013.

[23] J. Liouville. "Premier Memoire sur la détermination des intégrales dont la valeur est algébrique". Journ. Ec. Polyt. 14 (22. cahier) (1833), 124-148; Mem. Savans Etrangers Acad. Sci. Paris 5 (1838), 76-102.http: //books . google. es/books? id=08cAAAAAYAAJ\&printsec= frontcover\&hl=es\#v=onepage $\& q \& f=f a l$ se.Consultada Agosto 3, 2013.

[24] B. Terelius. "Symbolic Integration." Master"s Thesis.KHT. Sweden. 2009. http://www. nada. kth.se/utbildning/grukth/exjobb/rapportlistor/2009/rapporter09/terelius_ bjorn_09095.pdf. Consultada Agosto 3, 2013.

[25] E. Horowitz. "Algorithms for partial fraction decomposition and rational function integration." In Proceedings of SYMSAM"71, pages 441-457. ACM Press, 1971. http://research.cs.wisc . edu/techreports/1970/TR91. pdf. Consultada Agosto 3, 2013.

[26] M. Rothstein. "A new algorithm for the integration of exponential and logarithmic functions". Proceedings of the 1977 MACSYMA Users Conference, pages 263-274. NASA Pub. CP-2012, 1977.

[27] B. Buchberger et all. Computer Algebra. Simbolic and Algebraic Computation. 2nd Edition. SpingerVerlag. 1982.

¿Se puede saber si una función tiene primitiva elemental? . Walter Mora F.

Derechos Reservados @ 2015 Revista digital Matemática, Educación e Internet (http://tecdigital.tec.ac.cr/revistamatematica/) 